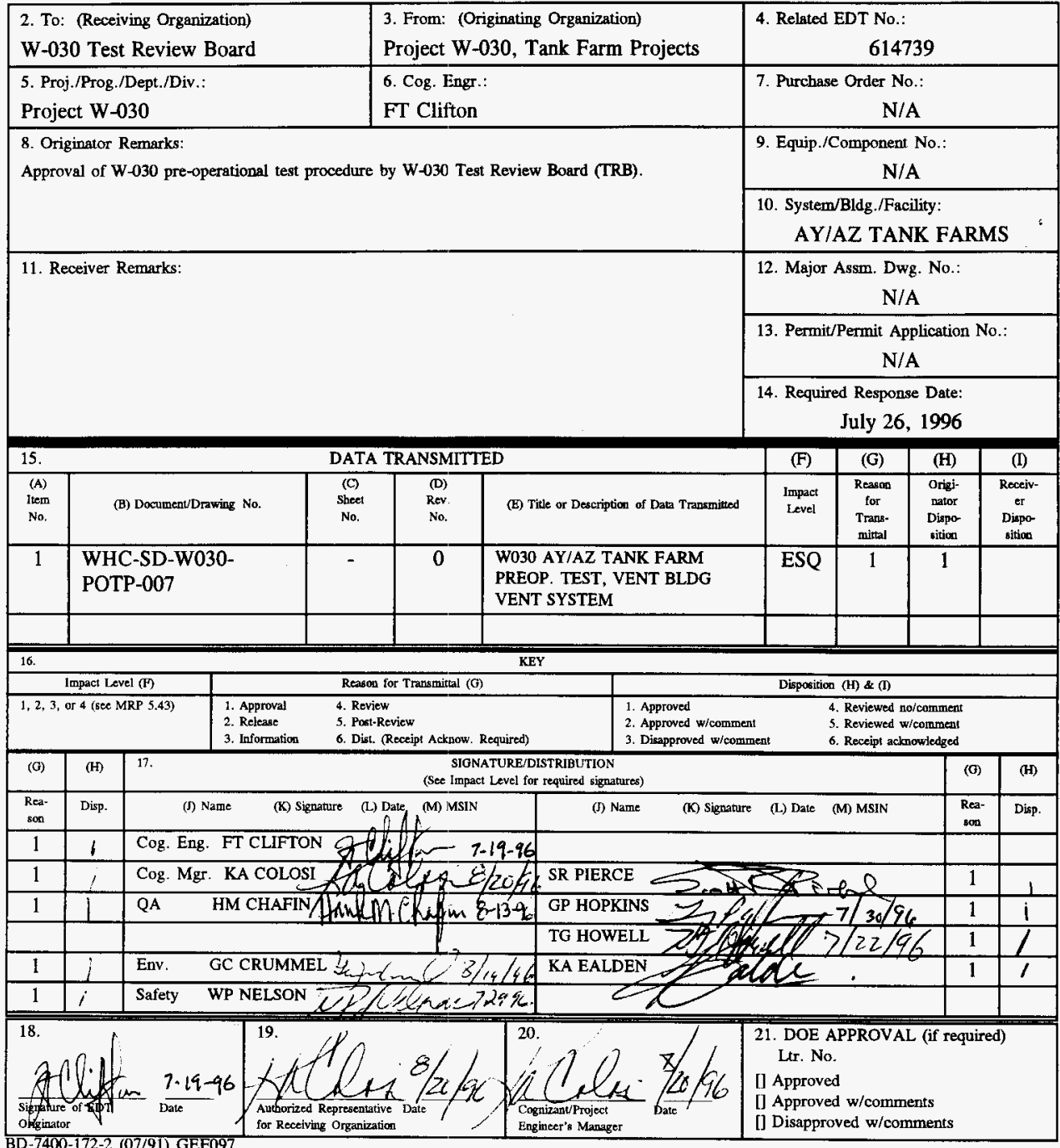

BD-7400-172-2 (07/91) GEF097 
WHC-SD-W030-POTP-007, Rev. 0

\title{
PREOPERATIONAL TEST, VENT BUILDING VENTILATION SYSTEM
}

\author{
FT Clifton
}

Westinghouse Hanford Company, Richland, WA 99352

U.S. Department of Energy Contract DE-AC06-87RL10930

$\begin{array}{lll}\text { EDT/ECN: } & 616340 & \text { UC: } 2030 \\ \text { Org Code: } & 8 K 240 & \text { Charge Code: NK201 } \\ \text { B\&R Code: } & \text { EW3130010 } & \text { Total Pages: } 76\end{array}$

Key Words: PROJECT W-030, TEST, VENTILATION, COOLING, UPGRADE

Abstract: Preoperational Test Procedure for Vent Building Ventilation System, Project $W-030$. Project $W-030$ provides a ventilation upgrade for the four Aging Waste Facillity tanks.

The Vent Building ventilation system provides ventilation, heating, cooling, and zone confinement control for the $W-030$ Project Vent Building.

The tests verify correct system operation and correct indications displayed by the central Monitor and Control system.

TRADEMARK DISCLAIMER. Reference herein to any specific commercial product, process, or service by trade name, trademark, manufacturer "or otherwise, does not necessarily constitute or imply its endorsement, recomendation, or favoring by the United States Government or any agency thereof or its contractors or subcontractors.

Printed in the United States of America. To obtain copies of this document, contact: WHC/BCS

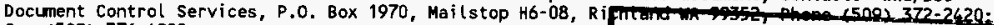
Fax (509) 376-4989.
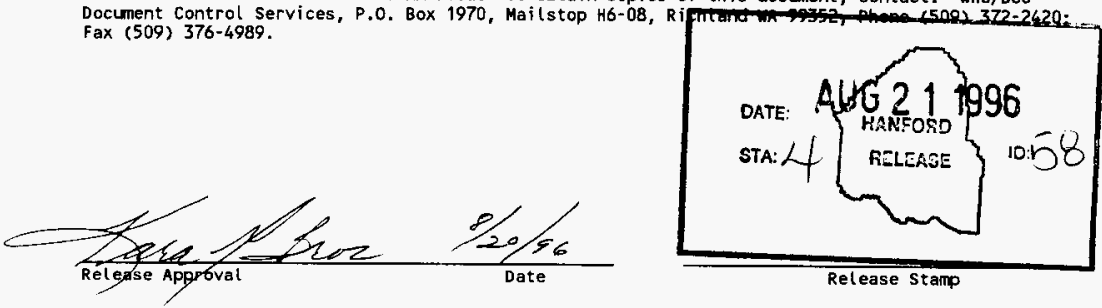

\section{Approved for Public Release}


PREOPERATIONAL TESTING, VENTILATION BUILDING VENTILATION SYSTEM

Revision No.

PAGE 1 OF 11

Effective Date

Author/Cognizant Engineer

T.G. HOWELL TZY

Print Name/Signature

APPROVAL DESIGNATOR So

PROCEDURE APPROVAL BY TEST REVIEW BOARD (TRB)

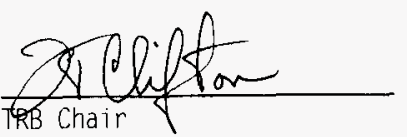

$\frac{7-19-96}{\text { Date }}$

TWRS Operations

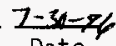

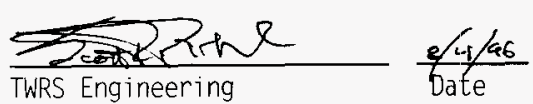
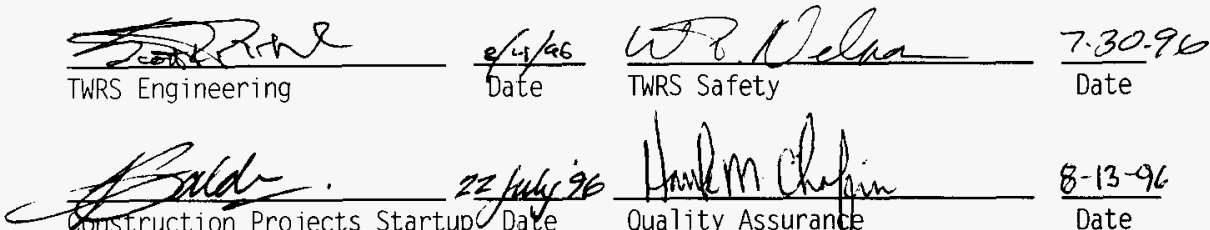

$2 z$ fuly $q 6$

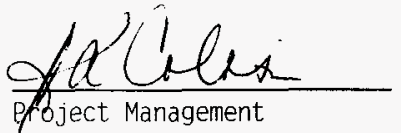

$\frac{8 / 20 / 96}{\text { Date }}$

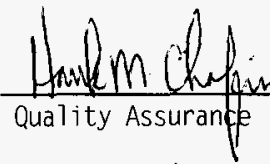

$\frac{8-13-96}{\text { Date }}$

the original signatures are on file. 


\section{PREOPERATIONAL TESTING, VENTILATION BUILDING VENTILATION SYSTEM WHC-SD-W030-POTP-007 \\ Revision No. \\ Effective Date \\ PAGE 2 OF 11}

\section{TABLE OF CONTENTS PAGE}

1.0 PURPOSE

2.0 INFORMATION

2.1 SCOPE

2.2 TERMS AND DEFINITIONS

2.3 RESPONSIBILITIES

2.4 CHANGE CONTROL

2.5 EXCEPTIONS

2.6 REFERENCES

2.7 ENVIRONMENTAL

2.8 SAFETY

2.9 RADIATION AND CONTAMINATION CONTROL

2.10 QUALITY ASSURANCE

2.11 GENERAL INFORMATION

2.12 LIMITS AND PRECAUTIONS

3.0 RECORDS

4.0 PREREQUISITES

5.0 PROCEDURE

ATTACHMENTS:

Attachment A - VENT BUILDING ACT TRAIN A DAMPER TEST PROCEDURE

Attachment B - VENT BUILDING TRAIN A EXHAUST FAN TEST PROCEDURE

Attachment $\mathrm{C}$ - VENT BUILDING ACT TRAIN A DIFFERENTIAL PRESSURE MONITORING

TEST PROCEDURE

Attachment D - VENT BUILDING ACT TRAIN B DAMPER TEST PROCEDURE

Attachment $E$ - VENT BUILDING TRAIN B EXHAUST FAN TEST PROCEDURE

Attachment F - VENT BUILDING TRAIN B DIFFERENTIAL PRESSURE MONITORING

TEST PROCEDURE

Attachment $G$ - VENT BUILDING AIR CONDITIONING UNIT TEST PROCEURE

Attachment $H$ - VENT BUILDING ROOM PRESSURE MONITORING AND CONTROL TEST PROCEDURE

Attachment I - VENT BUILDING VENTILATION STACK MONITOR TEST PROCEDURE

Attachment $\mathrm{J}$ - SIGNATURE/INITIAL VERIFICATION

Attachment $K$ - TEST LOG

Attachment $L$ - TEST EXCEPTION REPORT

Attachment $M$ - TEST EXCEPTION LOG 


\section{PREOPERATIONAL TESTING, VENTILATION BUILDING VENTILATION SYSTEM \\ WHC-SD-W030-POTP-007

Revision No.

Effective Date

1.0 PURPOSE

1.1 This procedure has been prepared to verify the Ventilation Building Ventilation System and the Stack Monitor System operates in accordance with system design.

\subsection{INFORMATION}

$2.1 \quad$ SCOPE

2.1.1 This procedure will demonstrate the operation of the following in the Ventilation Building Ventilation System:

- Exhaust Train controls and monitoring, filter pressure differential monitoring and alarms, damper controls and interlocks, and fan controls. interlocks and performance.

- Vent Building air conditioning unit operation, Vent Building room pressure monitoring and controls.

- Vent Building ventilation stack monitor operation. controls and alarms.

2.1.2 The Ventilation Building Ventilation system and the Stack Monitor System interface with each other and the Monitoring and Control System.

\subsubsection{This procedure wi11 demonstrate the operation of system} interlocks and controls both local and remote as applicable.

\subsection{TERMS AND DEFINITIONS}

2.2.1 HS - Hand Switch

2.2.2 LOI - Local Operator Interface device

2.2.3 MCS - Monitoring Control system

2.2.4 PCL - Process Logic Control

2.2.5 PI - Pressure Indicator

2.2.6 POTP - Preoperational Testing Procedure 


\section{PREOPERATIONAL TESTING, VENTILATION BUILDING VENTILATION SYSTEM WHC-SD-W030-POTP-007}

Revision No. 0

Effective Date

\subsection{RESPONSIBILITIES}

2.3.1 The Craft (TWRS Maintenance and/or Construction Forces) personnel are responsible for:

- $\quad$ Providing assistance during POPT testing.

- At least one technician must be a qualified "source user" per WHC-CM-4-14, part 1, section 2.3, to support this test.

\subsubsection{Test Director (TD) responsibilities:}

- $\quad$ Provides the equipment found in STEP 4.8 of this POTP.

- Safe and productive accomplishment of the tests necessary to achieve startup.

- $\quad$ Ensure safe working conditions and practices.

- $\quad$ Ensure compliance with test documents, Operational Safety Requirements/Documents (OSRS/OSDS) during testing.

- Communicate and coordinate the tests with the East Tank Farm Shift Manager.

- $\quad$ Ensure appropriate review/approval of any modifications to test procedures are completed prior to returning to work

- Direct line of communication and centralized point of control during normal, abnorma1, and casualty situations.

- $\quad$ Conducts pre-job planning meeting as necessary.

- Scheduling/rescheduling of the test as required.

- Delegates any of the above responsibilities as needed to a deputy. 
PREOPERATIONAL TESTING, VENTILATION BUILDING VENTILATION SYSTEM WHC-SD-W030-POTP-007

Revision No. $\underline{0}$

Effective Date

2.3.3 The Test Engineering Personnel (TEP) responsibilities:

- Providing technical support during testing.

- Providing programming support during testing.

- Forcing data in PLC program during testing.

- Direct preoperational testing

- Review test documents to validate acceptance

- Prepare post testing documents

- Records equipment status and data per this procedure.

- Conducts pre-job system walkdown.

2.3.4 Operations Personnel (OP) responsibilities:

- Observing test activities for training purposes.

2.3.5 Quality Control (QC) responsibilities:

- Witnessing the performance of the test procedure.

2.4 CHANGE CONTROL

2.4.1 Test procedure administrative or editorial changes required during testing may be accommodated as exceptions in the released test report. if the changes wi 11 not affect operating facility safety. function. or performance and will not compromise or influence test data. Requirement changes. changes to acceptance criteria, or changes to Danger. Caution. Special Precautions, or other safety or evironmenta 1 instructions in test procedures prepared as supporting documents must be made by engineering change notice. 
Revision No.

0

2.5 EXCEPTIONS

2.5.1 Exceptions to results or to the test procedure will be given a sequential number and recorded on Attachment $M$. Test

Exception log sheet. A test exception report, Attachment $L$. will be filled out to record and disposition each test exception.

2.6 REFERENCES

2.6.1 The following documents were used to write or are referenced in this procedure:

- $\quad$ Project W-030 Startup Test PTan Rev 0. WHC-SD-W030-SUP-003

- H-2-131073 Rev 1. Sht 1 and 2, A2702 Vent B1dg. Vent Sys.

- H-2-131077-1. Rev. 1. Sht 1 of 1, AZ702 Vent B1dg. Vent Sys.

- H-2-131081 Rev 1, Sht 1 of 1, AZ702 Vent B1dg. Vent Sys.

- Tank Farm Ventilation Upgrade, W-030-C2

- Tank Farm Ventilation Upgrade. W-030-C3

- W-030-P04

\subsection{ENVIRONMENTAL}

2.7.1 Hazardous and mixed waste should be disposed of according to T0-100-052, or by calling Environmental Waste Operations at 372-1208. 


\section{PREOPERATIONAL TESTING, VENTILATION BUILDING VENTILATION SYSTEM \\ Revision No. \\ Effective Date}

2.8 SAFETY

Warning - In addition to contamination hazards, operators should be aware of the possibility of coming into contact with poisonous snakes and spiders.

2.8.1 The following administrative procedures control work performed in this POTP:

- Safety Manua 1. WHC-CM-1-10

- Industrial Hygiene Manual, WHC-CM-1-11.

- Tank Farm Health and Safety Plan. (HASP) WHC-SD-WM-HSP-002

2.9 RADIATION AND CONTAMINATION CONTROL

2.9.1 The work covered by this procedure is performed outside of the tank farm and does not require entry into a radiation/contamination control area.

2.9.2 when using the check sources, follow the precautions listed in the "source user briefing" sheet.

2.10 QUALITY ASSURANCE

2.10.1 № Quality Assurance witness, holdpoints or verifications are required in this procedure. Quality Assurance sha11 review and approve the test procedure, the final test report and the disposition of all test exceptions.

2.11 GENERAL INFORMATION

2.11.1 None 
PREOPERATIONAL TESTING, VENTILATION BUILDING VENTILATION SYSTEM

Revision No.

PAGE 8 OF 11

Effective Date

\subsection{LIMITS AND PRECAUTIONS}

2.12.1 If during performance of this procedure, any of the following conditions are found, immediately notify the assigned TD and TEP:

- Any equipment malfunction which could prevent fulfillment of it's functional requirements.

- Personnel error or procedural inadequacy which could prevent fulfillment of procedural requirements.

2.12.2 The TD and TEP may choose to stop work and place equipment in a safe condition based on the significance of the malfunction, error or inadequacy.

2.12.3 Contact TD and TEP for additional instructions if changing plant conditions affect work or delays in work extend past end of the (testing) shift.

2.12.4 If any waste is generated during performance of this instruction consult Facility/Plant/Area Hazardous Waste Coordinator for specific instructions to ensure compliance with WHC and DOE environmental standards. as applicable, for correct disposal.

2.12.5 Comply with WHC and plant/facility specific lock and tag or over-tagging requirements, as applicable. 
PREOPERATIONAL TESTING, VENTILATION BUILDING VENTILATION SYSTEM WHC-SD-W030-POTP-007

Revision No.

0

PAGE 9 OF 11

Effective Date

2.12.6 A11 Measuring and Test Equipment (M\&TE) used during performance of this procedure to collect qualitative data with the exception of "timing devices", shall meet the following requirements:

- Be within its current calibration cycle as evidenced by an affixed calibration label.

- $\quad$ Be capable of the desired range.

- Have an accuracy (consistent with state-of-theart limitations) equal to or greater than the accuracy specified in the procedure.

2.12.7 Timing measurements shal 1 be made with commercially available timing devices.

2.12.8 The Test Engineer has overall control of the testing process and change record authorization for this POTP. The Test Engineer is responsible for conducting the test, data collection, and ensuring compliance with al1 POTP requirements.

2.12.9 All test data readings are to be taken and recorded for each location where the capability exists (i.e. local instrument, LOI, OS).

\section{$3.0 \quad$ RECORDS}

3.1 This POTP as well as a11 completed attachments/appendices will be filed as a permanent test record. 
PREOPERATIONAL TESTING, VE'NTILATION BUILDING VENTILATION SYSTEM

WHC-SD-W030-POTP-007

Revision No.

PAGE 10 OF 11

Effective Date

\subsection{PREREQUISITES}

NOTE: Unless otherwise specified, prerequisite actions may be performed in any order.

4.1 Perform a pretest briefing for al1 personnel involved in the performance of this POTP.

Test Director

4.2 Perform a walkdown inspection of the systems tested by this POTP. Test Engineer

4.3 Communication between the control room and equipment locations has been verified.

Test Director

4.4 The official POTP copy and a1l other photocopies that will be used during testing have been verified to be the latest approved/released document revision.

Test Director

4.5 The Test Engineer has verified, by reviewing the TAG LOGBOOK and walkdown of RWS systems being tested, that al1 components within and including the test boundary have been "BLUE" tagged.

Test Engineer

4.6 All open items have been evaluated and verified to not affect the POTP performance (Quality Assurance Nonconformance Reports <NCRS>. Construction Punch Lists, outstanding Engineering Change Notices $<$ ECNs>. Startup-originated Design Change Requests <CRs>. Test Deficiency Reports, and Master System Punch List items).

Test Director

4.7 All personnel who will be involved with this procedure have provided the required signature verification information in Attachment $\mathrm{J}$.

Test Director

WHC-SD-W030-POTP-007 


\section{PREOPERATIONAL TESTING, VENTILATION BUILDING VENTILATION SYSTEM WHC-SD-W030-POTP-007 \\ Revision No.

4.8 Verify that the vent cell roof hatch has be closed and sealed.

$$
\text { Test Director }
$$

4.9 EQUTPMENT/INSTRUMENTS

4.9.1 Digital Multi Meter

Manufacturer:

Serial No.

Model No

Calibration Due Date Calibration Date

4.9.2 Checkout the "check sources" from the HPT office when needed for performing the portion of this test applicable to the Beta Monitor.

\subsection{PROCEDURE}

5.1 The Vent Building Ventilation System testing shall be performed using Attachments $\mathrm{A}$ through $\mathrm{H}$ of this procedure.

5.2 The Vent Building Stack Monitor System testing shal1 be performed using Attachment I of this procedure. 
PREOPERATIONAL TESTING - VENTILATION BUILDING VENTILATION SYSTEM

\section{ATTACHMENT A}

INITIAL CONDITIONS

1.1 VERIFY/PERFORM the electrical line-up detailed in Appendix A.

Test Engineer Date

1.2 VERIFY/PERFORM the valve/damper line-up in Appendix B.

Test Engineer Date

2.1 SELECT Damper MK-AZ702K3-2A NORMAL on MCS graphic 20BldExh.v.

2.2 SELECT Damper MK-AZ702K3-2A AUTO on MCS graphic 20BldExh.v.

2.3 SELECT Damper MK-AZ702K3-2A OPEN on MCS graphic 20BldExh.v.

2.4 VERIFY the following:

- Damper MK-AZ702K3-2A stays CLOSED.

- $\quad$ MCS indicates CLOSED for Damper MK-AZ702K3-2A

Test Engineer Date

2.5 SELECT Damper MK-AZ702K3-2A MANUAL on MCS graphic 20BldExh.v.

2.6 SELECT Damper MK-AZ702K3-2A OPEN on MCS graphic 20BldExh.v.

2.7 VERIFY the following:

- Damper MK-AZ702K3-2A OPENS.

- $\quad$ MCS indicates OPEN for Damper MK-AZ702K3-2A

Test Engineer Date

2.8 SIMULATE a pressure differential $>1.5$ " w.c. at PDE-AZ702K303-1A.

2.9 VERIFY that Damper MK-AZ702K3-2A stays OPEN.

Test Engineer Date 
2.10 REMOVE simulation from PDE-AZ702K303-1A.

2.11 SIMULATE a pressure differential $>5$ " w.c. at PDE-AZ702K304-1A.

2.12 VERIFY that Damper MK-AZ702K3-2A stays OPEN.

Test Engineer Date

2.13 REMOVE simulation from PDE-AZ702K304-1A.

2.14 SIMULATE a high radiation level at RE-AZ702K3-1.

2.15 VERIFY that Damper MK-AZ702K3-2A stays OPEN.

Test Engineer Date

2.16 SELECT Damper MK-AZ702K3-2B NORMAL on MCS graphic 20BldExh.v.

2.17 SELECT Damper MK-AZ702K3-2B MANUAL on MCS graphic 20BIdExh.v.

2.18 SELECT Damper MK-AZ702K3-2B OPEN on MCS graphic 20BldExh.v.

2.19 SELECT Exhaust fan AZ702-K3-5-1B NORMAL on MCS graphic 20BldExh.v.

2.20 SELECT Exhaust fan AZ702-K3-5-1B MANUAL on MCS graphic 20BldExh.v.

2.21 SELECT Exhaust fan AZ702-K3-5-1B START on MCS graphic 20BldExh.v.

2.22 VERIFY the following:

- Damper MK-AZ702K3-2A CLOSES.

- MCS indicates CLOSED for Damper MK-AZ702K3-2A

Test Engineer Date

2.23 REMOVE simulation from RE-AZ702K3-1.

2.24 SELECT exhaust fan AZ702-K3-5-1B MANUAL on MCS graphic 20BldExh.v.

2.25 SELECT exhaust fan AZ702-K3-5-1B STOP on MCS graphic 20BldExh.v.

2.26 SELECT Damper MK-AZ702K3-2B MANUAL on MCS graphic 20BIdExh.v. 
2.27 SELECT Damper MK-AZ702K3-2B CLOSE on MCS graphic 20BldExh.v.

2.28 OPEN Damper MK-AZ702K3-2A by selecting OPEN on MCS graphic 20BldExh.v.

2.29 SIMULATE a pressure differential $>1.5$ " w.c. at PDE-AZ702K303-1A.

2.30 SELECT Damper MK-AZ702K3-2B MANUAL on MCS graphic 20BldExh.v.

2.31 SELECT Damper MK-AZ702K3-2B OPEN on MCS graphic 20BIdExh.v.

2.32 SELECT exhaust fan AZ702-K3-5-1B MANUAL on MCS graphic 20BIdExh.v.

2.33 SELECT exhaust fan AZ702-K3-5-1B START on MCS graphic 20BldExh.v.

2.34 VERIFY the following:

- Damper MK-AZ702K3-2A CLOSES.

- $\quad$ MCS indicates CLOSED for Damper MK-AZ702K3-2A

Test Engineer Date

2.35 REMOVE simulation from PDE-AZ702K303-1A.

2.36 SELECT exhaust fan AZ702-K3-5-1B MANUAL on MCS graphic 20BldExh.v.

2.37 SELECT exhaust fan AZ702-K3-5-1B STOP on MCS graphic 20BldExh.v.

2.38 SELECT Damper MK-AZ702K3-2B MANUAL on MCS graphic 20BldExh.v.

2.39 SELECT Damper MK-AZ702K3-2B CLOSE on MCS graphic 20BldExh.v.

2.40 OPEN Damper MK-AZZ702K3-2A by selecting OPEN on MCS graphic 20BIdExh.v.

2.41 SIMULATE a pressure differential $>5$ " w.c. at PDE-AZ702K304-1A.

2.42 SELECT Damper MK-AZ702K3-2B MANUAL on MCS graphic 20BldExh.v.

2.43 SELECT Damper MK-AZ702K3-2B OPEN on MCS graphic 20BldExh.v.

2.44 SELECT exhaust fan AZ702-K3-5-1B MANUAL on MCS graphic 20BldExh.v.

2.45 SELECT exhaust fan AZ702-K3-5-1B START on MCS graphic 20BIdExh.v. 
2.46 VERIFY the following:

- Damper MK-AZ702K3-2A CLOSES.

- $\quad$ MCS indicates CLOSED for Damper MK-AZ702K3-2A

Test Engineer Date

2.47 REMOVE simulation from PDE-AZ702K304-1A.

2.48 SELECT exhaust fan AZ702-K3-5-1B STOP on MCS graphic 20BIdExh.v.

2.49 SELECT Damper MK-AZ702K3-2B CLOSE on MCS graphic 20BldExh.v.

2.50 SELECT Damper MK-AZ702K3-2A CLOSE on MCS graphic 20BldExh.v.

2.51 SELECT Damper MK-AZ702K3-2A AUTO on MCS graphic 20BldExh.v. 
PREOPERATIONAL TESTING - VENTILATION BUILDING VENTILATION SYSTEM

Revision No. 0.

\section{Appendix A}

\section{Electrical Line-up}

\begin{tabular}{|c|c|c|}
\hline $\begin{array}{l}\text { BREAKDESWIICH } \\
\text { NOE. }\end{array}$ & BREAKERSSWITCHYOCATION & REQUIREA POSTMON \\
\hline 1 & $\begin{array}{l}\text { Vent Bldg. Exhaust Fan AZ702-K3-5-1A } \\
\text { in Panelboard PP- } 16 \text { located in E/I Room }\end{array}$ & CLOSED \\
\hline 2 & $\begin{array}{l}\text { Vent Facility Panelboard PP- } 5 \text { in E/I } \\
\text { Room }\end{array}$ & CLOSED \\
\hline 3 & $\begin{array}{l}\text { Vent Bldg. Air Conditioning Unit AZ702- } \\
\text { K2-6-1 in Panelboard } 16 \text { in E/ Room. }\end{array}$ & CLOSED \\
\hline 4 & $\begin{array}{l}\text { Vent Bldg. Exhaust Fan AZ702-K3-5-1B } \\
\text { in Panelboard PP-16 located in E/I Room }\end{array}$ & CLOSED \\
\hline
\end{tabular}


PREOPERATIONAL TESTING - VENTILATION BUILDING VENTILATION SYSTEM

Revision No. 0.

WHC-SD-W030-POTP-007

Page 6 of 7.

ATTACHMENT A - VENT BUILDING ACT TRAIN A DAMPER TEST PROCEDURE

\section{Appendix B}

\section{Damper/Valve Line-up}

\begin{tabular}{|c|c|c|}
\hline DAMPER/VALVE NO. & LOCATION & REQUIRED POSITION \\
\hline MK-AZ702K3-1A & $\begin{array}{l}\text { Filter Train A inlet isolation damper } \\
\text { located in building filter room. }\end{array}$ & OPEN \\
\hline MK-AZ702K305-1A & $\begin{array}{c}\text { Exhaust Fan AZ702-K3-5-1A } \\
\text { Discharge Damper located in } \\
\text { building filter room. }\end{array}$ & OPEN \\
\hline 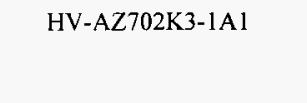 & $\begin{array}{c}\text { PDE-AZ702K303-1A Isolation } \\
\text { valve located in building filter } \\
\text { room. }\end{array}$ & OPEN \\
\hline $\mathrm{HV}-\mathrm{AZ702K} 3-1 \mathrm{~A} 2$ & $\begin{array}{l}\text { PDE-AZ702K303-1 A/PDE- } \\
\text { AZ702K304-1 A Isolation valve } \\
\text { located in building filter room. }\end{array}$ & OPEN \\
\hline HV-AZ702K3-1A3 & $\begin{array}{c}\text { PDE-AZ702K304-1A Isolation } \\
\text { valve located in building filter } \\
\text { room. }\end{array}$ & OPEN \\
\hline MK-AZ702K3-1B & $\begin{array}{l}\text { Filter Train B inlet isolation damper } \\
\text { located in building filter room. }\end{array}$ & OPEN \\
\hline MK-AZ702K305-1B & $\begin{array}{c}\text { Exhaust Fan AZ702-K3-5-1B } \\
\text { Discharge Damper located in } \\
\text { building filter room. }\end{array}$ & OPEN \\
\hline 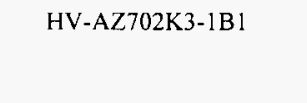 & $\begin{array}{c}\text { PDE-AZ702K303-1B Isolation } \\
\text { valve located in building filter } \\
\text { room. }\end{array}$ & OPEN \\
\hline $\mathrm{HV}-\mathrm{AZ} 702 \mathrm{~K} 3-1 \mathrm{~B} 2$ & $\begin{array}{l}\text { PDE-AZ702K303-1 B/PDE- } \\
\text { AZ702K 304-1B Isolation valve } \\
\text { located in building filter room. }\end{array}$ & OPEN \\
\hline HV-AZ702K3-1B3 & $\begin{array}{l}\text { PDE-AZ702K304-1B Isolation } \\
\text { valve located in building filter } \\
\text { room. }\end{array}$ & OPEN \\
\hline HV-AZ702K2-1A & $\begin{array}{l}\text { PDE-AZ702K2-1 Isolation valve } \\
\text { located in Stack Monitor room. }\end{array}$ & OPEN \\
\hline $\mathrm{HV}-\mathrm{AZ} 702 \mathrm{~K} 2-2 \mathrm{~A}$ & $\begin{array}{l}\text { PDE-AZ702K2-2 Isolation valve } \\
\text { located in Stack Monitor room. }\end{array}$ & OPEN \\
\hline
\end{tabular}


PREOPERATIONAL TESTING - VENTILATION BUILDING VENTILATION SYSTEM

Revision No. 0.

WHC-SD-W030-POTP-007

Page 7 of 7.

ATTACHMENT A - VENT BUILDING ACT TRAIN A DAMPER TEST PROCEDURE

\begin{tabular}{|c|c|c|}
\hline DAMPER/VALVE NO. & LOCATION & REQUIRED POSITION \\
\hline HV-AZ702K2-3A & $\begin{array}{c}\text { PDE-AZ702K2-3 Isolation valve } \\
\text { located in Stack Monitor room. }\end{array}$ & OPEN \\
\hline HV-AZ702K3-1A & $\begin{array}{c}\text { PDE-AZ702K3-1 Isolation valve } \\
\text { located in Stack Monitor room. }\end{array}$ & OPEN \\
\hline HV-AZ702K3-1B & $\begin{array}{c}\text { PDE-AZ702K3-1 Isolation } \\
\text { valvelocated in Filter Room B. }\end{array}$ & OPEN \\
\hline HV-AZ702K204-1A & $\begin{array}{c}\text { PDE-AZ702K204-1 Isolation valve } \\
\text { located in Primary Vent Filter Room } \\
\text { A. }\end{array}$ & OPEN \\
\hline HV-AZ702K204-1B & $\begin{array}{c}\text { PDE-AZ702K204-1 Isolation valve } \\
\text { located in Primary Vent Filter Room } \\
\text { A. }\end{array}$ & OPEN \\
\hline HV-AZ702K204-2A & $\begin{array}{c}\text { PDE-AZ702K204-2 Isolation valve } \\
\text { located in Primary Vent Filter Room } \\
\text { B. }\end{array}$ & OPEN \\
\hline HV-AZ702K204-1B & $\begin{array}{c}\text { PDE-AZ702K204-2 Isolation valve } \\
\text { located in Primary Vent Filter Room } \\
\text { B. }\end{array}$ & \\
\hline & & \\
\hline
\end{tabular}




\section{ATTACHMENT B}

INITIAL CONDITIONS

1.1 VERIFY/PERFORM the electrical line-up detailed in Attachment A, Appendix A.

Test Engineer Date

1.2 VERIFY/PERFORM the valve/damper line-up in Attachment A, Appendix B.

Test Engineer Date

\section{PROCEDURE}

2.1 Fan AZ702-K3-5-1A Control from Local control switch HS-AZ702K305-1A1.

2.1.1 OPEN manual damper MK-AZ702K3-1A.

Test Engineer Date

2.1.2 OPEN manual damper MK-AZ702K305-1A.

Test Engineer Date

2.1.3 VERIFY local control switch HS-AZ702K305-1A1 in the STOP position. Test Engineer Date

2.1.4 SET fan AZ702-K3-5-1A flow control FIC-702K3-3A to $925 \mathrm{scfm}$ on MCS graphic 20BldExh.v..

2.1.5 SELECT Damper MK-AZ702K3-2A MANUAL on MCS graphic 20BldExh.v.

2.1.6 SELECT Damper MK-AZ702K3-2A OPEN on MCS graphic 20BldExh.v.

2.1.7 SELECT Air Conditioning Unit AZ702-K2-6-1 AUTO on MCS graphic 20BldExh.v.

2.1.8 SELECT Exhaust Fan AZ702-K3-5-1A NORMAL on MCS graphic 20BldExh.v.

2.1.9 SELECT Exhaust Fan AZ702-K3-5-1A MANUAL on MCS graphic 20BldExh.v. 2.1.10 SELECT Exhaust Fan AZ702-K3-5-1A START on MCS graphic 20BldExh.v. 
2.1.11 VERIFY that fan AZ702-K3-5-1A does NOT START.

Test Engineer

Date

2.1.12 PLACE local control switch HS-AZ702K305-1A1 in the START position.

2.1.13 VERIFY the following:

2.1.13.1 Exhaust Fan AZ702-K3-5-1A STARTS.

2.1.13.2 Air Conditioning Unit AZ702-K2-6-1 STARTS.

Test Engineer

Date

2.1.14 After a stabilization period of 15 minutes RECORD the following data:

2.1.14.1 for exhaust fan AZ702-K3-5-1A:

Motor Current

Motor Voltage

Total Airflow

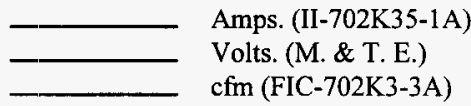

2.1.14.2 for air conditioning unit AZ702-K2-6-1:

Motor Current

Motor Voltage

Total Airflow

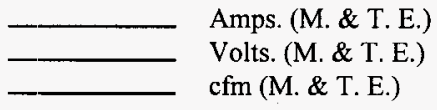

2.1.15 Allow exhaust fan AZ702-K3-5-1A to operate for sufficient length of time needed to obtain system air flow, vibration and bearing temperature data. RECORD on Exhibit A data sheets.

2.1.16 SELECT Exhaust Fan AZ702-K3-5-1A STOP on MCS graphic 20BldExh.v.

2.1.17 VERIFY that exhaust fan AZ702-K3-5-1A does NOT STOP.

Test Engineer Date

2.1.18 SIMULATE an air flow $<200 \mathrm{cfm}$ at FIT-702K3-3A.

2.1.19 VERIFY that exhaust fan AZ702-K3-5-1A does NOT STOP.

Test Engineer Date 
2.1.20 REMOVE simulation from FIT-702K3-3A .

Test Engineer Date

2.1.21 PLACE local control switch HS-AZ702K305-1A1 in the STOP position.

2.1.22 VERIFY the following:

2.1.22.1 Exhaust Fan AZ702-K3-5-1A STOPS.

2.1.22.2 Air Conditioning Unit AZ702-K2-6-1 STOPS.

Test Engineer Date

\subsection{Fan AZ702-K3-5-1A Control from MCS}

2.2.1 SELECT Damper MK-AZ702K3-2B AUTO on MCS graphic 20BldExh.v.

2.2.2 SELECT Exhaust Fan AZ702-K3-5-1B NORMAL on MCS graphic 20BldExh.v.

2.2.3 SELECT Exhaust Fan AZ702-K3-5-1B AUTO on MCS graphic 20BldExh.v.

2.2.4 SELECT Damper MK-AZ702K3-2A CLOSE on MCS graphic 20BIdExh.v.

2.2.5 SELECT Damper MK-AZ702K3-2A AUTO on MCS graphic 20BldExh.v.

2.2.6 PLACE the local handswitch HS-AZ702K305-1Al in REMOTE.

2.2.7 SELECT Exhaust Fan AZ702-K3-5-1A START on MCS graphic 20BldExh.v.

2.2.8 VERIFY the following:

2.2.8.1 Damper MK-AZ702K3-2A OPENS.

2.2.8.2 Fan AZ702-K3-5-1 A STARTS.

2.2.8.3 Air Conditioning Unit AZ702-K2-6-1 STARTS.

Test Engineer Date

2.2.9 SELECT Exhaust Fan AZ702-K3-5-1A OVERRIDE on MCS graphic 20BIdExh.v.

2.2.10 SIMULATE an air flow $<200 \mathrm{cfm}$ at FIT-702K3-3A. 
PREOPERATIONAL TESTING, VENTILATION BUILDING VENTILATION SYSTEM

Revision No. 0.

WHC-SD-W030-POTP-007

Page 4 of 6.

ATTACHMENT B - VENT BUILDING TRAIN A EXHAUST FAN TEST PROCEDURE

2.2.11 VERIFY that exhaust fan AZ702-K3-5-1A does NOT STOP.

Test Engineer

Date

2.2.12 SELECT Exhaust Fan AZ702-K3-5-1A NORMAL on MCS graphic 20BldExh.v.

2.2.13 VERIFY the following:

2.2.13.1 Exhaust fan AZ702-K3-5-1A STOPS.

2.2.13.2 Damper MK-AZ702K3-2A CLOSES.

2.2.13.3 Air Conditioning Unit AZ702-K2-6-1 STOPS.

2.2.13.4 Exhaust fan AZ702-K3-5-1B STARTS.

2.2.13.5 Damper MK-AZ702K3-2B OPENS.

2.2.13.6 Air Conditioning Unit AZ702-K2-6-1 RE-STARTS.

Test Engineer

Date

2.2.14 REMOVE simulation from FIT-702K3-3A.

Test Engineer

Date

2.2.15 SELECT exhaust fan AZ702-K3-5-1A AUTO on MCS graphic 20BldExh.v.

2.2.16 OPEN circuit breaker no. 4 in Panelboard PP-16 to SIMULATE failure of exhaust fan AZ702-K3-5-1B.

2.2.17 VERIFY the following:

2.2.17.1 Exhaust fan AZ702-K3-5-1B STOPS.

2.2.17.2 Damper MK-AZ702K3-2B CLOSES.

2.2.17.3 Air Conditioning Unit AZ702-K2-6-1 STOPS.

2.2.17.4 Exhaust fan AZ702-K3-5-1A STARTS.

2.2.17.5 Damper MK-AZ702K3-2A OPENS.

2.2.17.6 Air Conditioning Unit AZ702-K2-6-1 RE-STARTS.

Test Engineer Date

2.2.18 SELECT exhaust fan AZ702-K3-5-1A STOP on MCS graphic 20BldExh.v. 
2.2.19 VERIFY the following:

- exhaust fan AZ702-K3-5-1A STOPS.

- damper MK-AZ702K3-2A CLOSES.

Test Engineer

Date 


\section{PREOPERATIONAL TESTING, VENTILATION BUILDING VENTILATION SYSTEM \\ Revision No. 0. \\ WHC-SD-W030-POTP-007 \\ Page 6 of 6. \\ ATTACHMENT B - VENT BUILDING TRAIN A EXHAUST FAN TEST PROCEDURE}

\section{Exhibit A}

\begin{tabular}{|l|l|l|}
\hline System:Vent Bldg. Exhaust - Train A. & Fan No.: AZ702-K3-5-1A & Fan Type: Centrifugal \\
\hline Drive Type: Belt. & Service:Vent Building Ventilation & \\
\hline
\end{tabular}

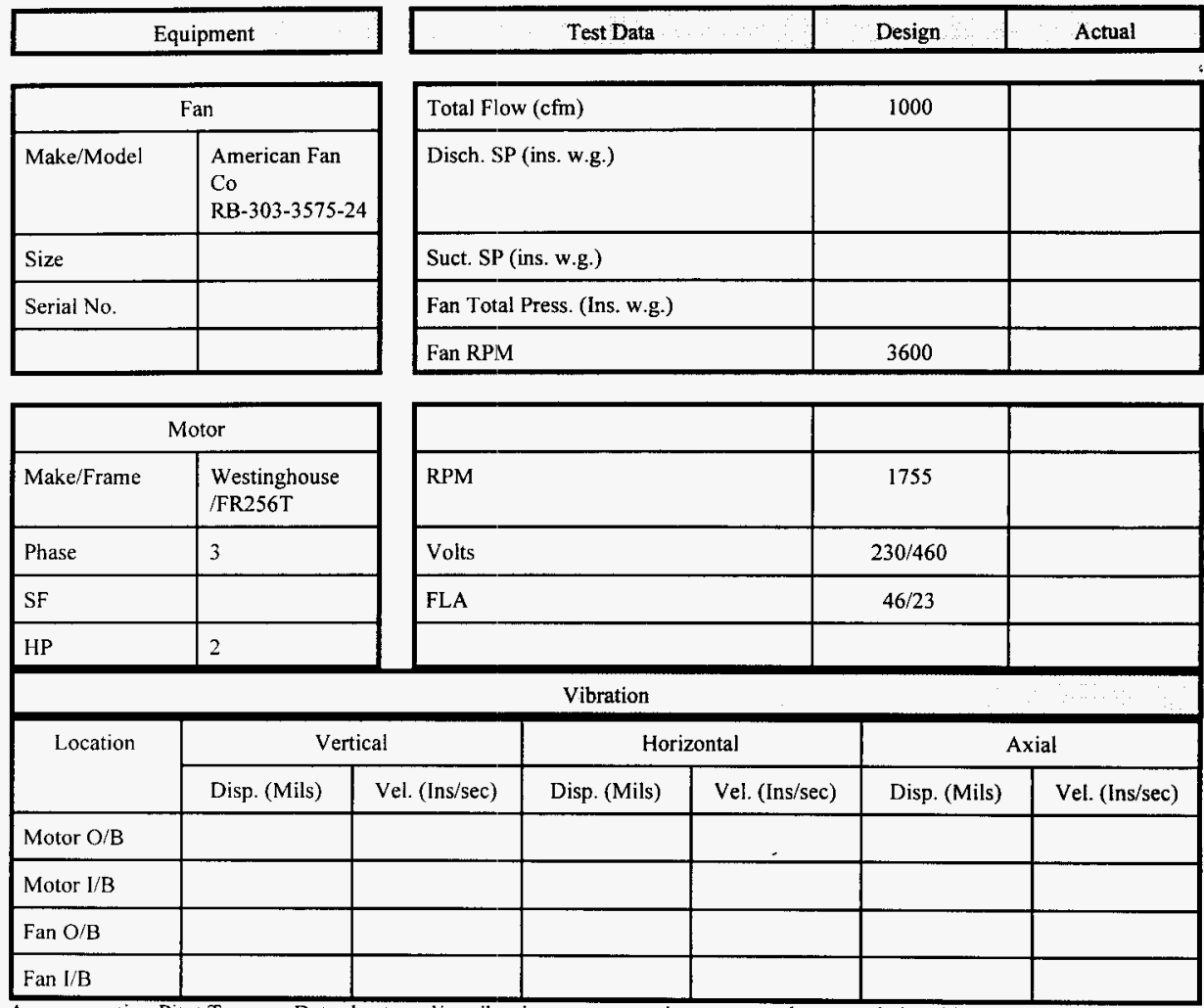

Any supporting Pitot Traverse Data sheets and/or vibration spectrum printouts are to be appended to this data sheet.

ROOM/FILTER PRESSURE DIFFERENTIALS (ins. w.c)

\begin{tabular}{|l|l|l|l|l|l|l|l|}
\hline PDI-702K2-1 & & PDI-702K2-7 & & PDI-702K24-2 & & PDI-702K34-1A & \\
\hline PDIC-702K2-2 & & PDI-702K2-8 & & PDIC-702K3-1 & & PDI-702K33-1B & \\
\hline PDIC-702K2-3 & & PDI-702K24-1 & & PDI-702K33- & & PDI-702K34-1B & \\
\hline
\end{tabular}


PREOPERATIONAL TESTING - VENTILATION BUILDING VENTILATION SYSTEM

Revision No. 0.

WHC-SD-W030-POTP-007

Page 1 of 2.

ATTACHMENT C- VENT BUILDING ACT TRAIN A DIFFERENTIAL PRESSURE MONITORING TEST PROCEDURE

\section{ATTACHMENT C}

\section{INITIAL CONDITIONS}

1.1 VERIFY/PERFORM the electrical line-up in Attachment A, Appendix A.

Test Engineer

Date

1.2 VERIFY/PERFORM the valve/damper line-up in Attachment A, Appendix B.

Test Engineer Date

1.3 OPEN manual damper MK-AZ702K3-1A.

Test Engineer

Date

1.4 OPEN manual damper MK-AZ702K305-1A.

Test Engineer Date

1.5 VERIFY local control switch HS-AZ702K305-1A1 in the REMOTE position.

Test Engineer Date

1.6 SET fan AZ702-K3-5-1A flow control FIC-702K3-3A960 scfm on MCS graphic 20BldExh.v.

1.7 SELECT Damper MK-AZ702K3-2A MANUAL on MCS graphic 20BldExh.v.

1.8 SELECT Damper MK-AZ702K3-2A OPEN on MCS graphic 20BldExh.v.

1.9 SELECT Air Conditioning Unit AZ702-K2-6-1 AUTO on MCS graphic 20BldExh.v.

1.10 SELECT exhaust fan AZ702-K3-5-1A NORMAL on MCS graphic 20BldExh.v.

1.11 SELECT exhaust fan AZ702-K3-5-1A MANUAL on MCS graphic 20BIdExh.v.

2 PROCEDURE

2.1 START the Building Vent System by selecting exhaust fan AZ702-K3-5-1A START command on MCS graphic 20BldExh.v. 
PREOPERATIONAL TESTING - VENTILATION BUILDING VENTILATION SYSTEM

Revision No. 0.

WHC-SD-W030-POTP-007

Page 2 of 2.

ATTACHMENT C- VENT BUILDING ACT TRAIN A DIFFERENTIAL PRESSURE MONITORING TEST PROCEDURE

2.2 WAIT 10 minutes for system to stabilize.

2.3 SIMULATE an increasing pressure differential at PDE-AZ702303-1A until PDAH-AZ702303-1A annunciates at the MCS.

2.4 RECORD pressure differential reading at PDI-AZ702303-1A.

PDI-AZ702303-1A Ins. w.c.(0.3 ins. w.c. expected).

Test Engineer Date

2.5 INCREASE pressure differential simulation at PDE-AZ702303-1A until PDAHH-AZ702303-1A annunciates at the MCS.

2.6 RECORD pressure differential reading at PDI-AZ702303-1A.

PDI-AZ702303-1A Ins. w.c.(0.5 ins. w.c. expected).

Test Engineer Date

2.7 REMOVE simulation from PDE-AZ702303-1A.

Test Engineer Date

2.8 SIMULATE an increasing pressure differential at PDE-AZ702304-1A until PDAH-AZ702304-1A annunciates at the MCS.

2.9 RECORD pressure differential reading at PDI-AZ702304-1A.

PDI-AZ702304-1A Ins. w.c.(3.0 ins. w.c. expected).

Test Engineer Date

2.10 INCREASE pressure differential simulation at PDE-AZ7702304-1A until PDAHH-AZ702304-1A annunciates at the MCS.

2.11 RECORD pressure differential reading at PDI-AZ702304-1A.

PDI-AZ702304-1A Ins. w.c.(5.0 ins. w.c. expected).

Test Engineer Date

2.12 REMOVE simulation from PDE-AZ702304-1A.

Test Engineer Date 
PREOPERATIONAL TESTING - VENTILATION BUILDING VENTILATION SYSTEM

Revision No. 0.

WHC-SD-W030-POTP-007

Page 1 of 4.

ATTACHMENT D - VENT BUILDING ACT TRAIN B DAMPER TEST PROCEDURE

\section{ATTACHMENT D}

INITIAL CONDITIONS

1.1 VERIFY/PERFORM the electrical line-up detailed in Attachment A, Appendix A.

Test Engineer

Date

1.2 VERIFY/PERFORM the valve/damper line-up in Attachment A, Appendix B.

Test Engineer

Date

PROCEDURE

2.1 SELECT Damper MK-AZ702K3-2B NORMAL on MCS graphic 20BldExh.v.

2.2 SELECT Damper MK-AZ702K3-2B AUTO on MCS graphic 20BldExh.v.

2.3 SELECT Damper MK-AZ702K3-2B OPEN on MCS graphic 20BldExh.v.

2.4 VERIFY the following:

- Damper MK-AZ702K3-2B stays CLOSED.

- $\quad$ MCS indicates CLOSED for Damper MK-AZ702K3-2B

Test Engineer Date

2.5 SELECT Damper MK-AZ702K3-2B MANUAL on MCS graphic 20BldExh.v.

2.6 SELECT Damper MK-AZ702K3-2B OPEN on MCS graphic 20BldExh.v.

2.7 VERIFY the following:

- Damper MK-AZ702K3-2B OPENS.

- $\quad$ MCS indicates OPEN for Damper MK-AZ702K3-2B

Test Engineer Date

2.8 SIMULATE a pressure differential >1.5" w.c. at PDE-AZ702K303-1B.

2.9 VERIFY that Damper MK-AZ702K3-2B stays OPEN.

Test Engineer Date 
2.10 REMOVE simulation from PDE-AZ702K303-1B.

2.11 SIMULATE a pressure differential >5" w.c. at PDE-AZ702K304-1B.

2.12 VERIFY that Damper MK-AZ702K3-2B stays OPEN.

Test Engineer Date

2.13 REMOVE simulation from PDE-AZ702K304-1B.

2.14 SIMULATE a high radiation level at RE-AZ702K3-1.

2.15 VERIFY that Damper MK-AZ702K3-2B stays OPEN.

Test Engineer Date

2.16 SELECT Damper MK-AZ702K3-2A NORMAL on MCS graphic 20BldExh.v.

2.17 SELECT Damper MK-AZ702K3-2A MANUAL on MCS graphic 20BldExh.v.

2.18 SELECT Damper MK-AZ702K3-2A OPEN on MCS graphic 20BIdExh.v.

2.19 SELECT Exhaust fan AZ702-K3-5-1B NORMAL on MCS graphic 20BldExh.v.

2.20 SELECT Exhaust fan AZ702-K3-5-1B MANUAL on MCS graphic 20BldExh.v.

2.21 SELECT Exhaust fan AZ702-K3-5-1B START on MCS graphic 20BldExh.v.

2.22 VERIFY the following:

- Damper MK-AZ702K3-2B CLOSES. -

- $\quad$ MCS indicates CLOSED for Damper MK-AZ702K3-2B

Test Engineer Date

2.23 REMOVE simulation from RE-AZ702K3-1.

2.24 SELECT exhaust fan AZ702-K3-5-1A MANUAL on MCS graphic 20BldExh.v.

2.25 SELECT exhaust fan AZ702-K3-5-1A STOP on MCS graphic 20BldExh.v.

2.26 SELECT Damper MK-AZ702K3-2A MANUAL on MCS graphic 20BldExh.v. 
PREOPERATIONAL TESTING - VENTILATION BUILDING VENTILATION SYSTEM

2.27 SELECT Damper MK-AZ702K3-2A CLOSE on MCS graphic 20BldExh.v.

2.28 OPEN Damper MK-AZ702K3-2B by selecting OPEN on MCS graphic 20BldExh.v.

2.29 SIMULATE a pressure differential $>1.5 "$ w.c. at PDE-AZ702K303-1B.

2.30 SELECT Damper MK-AZ702K3-2A MANUAL on MCS graphic 20BldExh.v.

2.31 SELECT Damper MK-AZ702K3-2A OPEN on MCS graphic 20BldExh.v.

2.32 SELECT exhaust fan AZ702-K3-5-1A MANUAL on MCS graphic 20BldExh.v.

2.33 SELECT exhaust fan AZ702-K3-5-1A START on MCS graphic 20BIdExh.v.

2.34 VERIFY the following:

- $\quad$ Damper MK-AZZ702K3-2B CLOSES.

- $\quad$ MCS indicates CLOSED for Damper MK-AZ702K3-2B

Test Engineer Date

2.35 REMOVE simulation from PDE-AZ702K303-1B.

2.36 SELECT exhaust fan AZ702-K3-5-1A MANUAL on MCS graphic 20BldExh.v.

2.37 SELECT exhaust fan AZ702-K3-5-1A STOP on MCS graphic 20BldExh.v.

2.38 SELECT Damper MK-AZ702K3-2A MANUAL on MCS graphic 20BldExh.v.

2.39 SELECT Damper MK-AZ702K3-2A CLOSE on MCS graphic 20BldExh.v.

2.40 OPEN Damper MK-AZ702K3-2B by selecting OPEN on MCS graphic 20BldExh.v.

2.41 SIMULATE a pressure differential >5" w.c. at PDE-AZ702K304-1B.

2.42 SELECT Damper MK-AZ702K3-2A MANUAL on MCS graphic 20BldExh.v.

2.43 SELECT Damper MK-AZ702K3-2A OPEN on MCS graphic 20BldExh.v.

2.44 SELECT exhaust fan AZ702-K3-5-1A MANUAL on MCS graphic 20BldExh.v.

2.45 SELECT exhaust fan AZ702-K3-5-1A START on MCS graphic 20BldExh.v. 
2.46 VERIFY the following:

- Damper MK-AZZ702K3-2B CLOSES.

- $\quad$ MCS indicates CLOSED for Damper MK-AZ702K3-2B

Test Engineer Date

2.47 REMOVE simulation from PDE-AZ702K304-1B.

2.48 SELECT exhaust fan AZ702-K3-5-1A STOP on MCS graphic 20BIdExh.v.

2.49 SELECT Damper MK-AZ702K3-2A CLOSE on MCS graphic 20BldExh.v.

2.50 SELECT Damper MK-AZ702K3-2B CLOSE on MCS graphic 20BldExh.v.

2.51 SELECT Damper MK-AZ702K3-2B AUTO on MCS graphic 20BldExh.v. 
PREOPERATIONAL TESTING, VENTILATION BUILDING VENTILATION SYSTEM

\section{ATTACHMENT E}

INITIAL CONDITIONS

1.1 VERIFY/PERFORM the electrical line-up detailed in Attachment A, Appendix A.

Test Engineer Date

1.2 VERIFY/PERFORM the valve/damper line-up in Attachment A, Appendix B.

Test Engineer Date

2.1 Fan AZ702-K3-5-1B Control from Local control switch HS-AZ702K305-1B1.

2.1.1 OPEN manual damper MK-AZ702K3-1B.

Test Engineer Date

2.1.2 OPEN manual damper MK-AZ702K305-1B.

Test Engineer Date

2.1.3 VERIFY local control switch HS-AZ702K305-1B1 in the STOP position.

Test Engineer Date

2.1.4 SET fan AZ702-K3-5-1B flow control FIC-702K3-3B to $925 \mathrm{scfm}$ on MCS graphic 20BldExh.v.

2.1.5 SELECT Damper MK-AZ702K3-2B MANUAL on MCS graphic 20BldExh.v.

2.1.6 SELECT Damper MK-AZ702K3-2B OPEN on MCS graphic 20BldExh.v.

2.1.7 SELECT Air Conditioning Unit AZ702-K2-6-1 AUTO on MCS graphic 20BldExh.v.

2.1.8 SELECT exhaust fan AZ702-K3-5-1B NORMAL on MCS graphic 20BldExh.v.

2.1.9 SELECT exhaust fan AZ702-K3-5-1B MANUAL on MCS graphic 20BldExh.v.

2.1.10 SELECT exhaust fan AZ702-K3-5-1B START on MCS graphic 20B1dExh.v. 
PREOPERATIONAL TESTING, VENTILATION BUILDING VENTILATION SYSTEM

Revision No. $0 . \quad$ WHC-SD-W030-POTP-007 $\quad$ Page 2 of 6.

ATTACHMENT E - VENT BUILDING TRAIN B EXHAUST FAN TEST PROCEDURE

2.1.11 VERIFY that fan AZ702-K3-5-1B does NOT START.

Test Engineer

Date

2.1.12 PLACE local control switch HS-AZ702K305-1B1 in the START position.

2.1.13 VERIFY the following:

2.1.13.1 Exhaust Fan AZ702-K3-5-1B STARTS.

2.1.13.2 Air Conditioning Unit AZ702-K2-6-1 STARTS.

Test Engineer

Date

2.1.14 After a stabilization period of 15 minutes RECORD the following data for exhaust fan AZ702-K3-5-1B:

Motor Current

Motor Voltage

Total Airflow

Amps. (II-702K35-1B)

Volts. (M. \& T. E.)

cfm (FIC-702K3-3)

2.1.15 Allow exhaust fan AZ702-K3-5-1B to operate for sufficient length of time needed to obtain system air flow, vibration and bearing temperature data. RECORD on Exhibit A data sheets.

2.1.16 SELECT Exhaust Fan AZ702-K3-5-1B STOP on MCS graphic 20BldExh.v.

2.1.17 VERIFY that exhaust fan AZ702-K3-5-1B does NOT STOP.

Test Engineer Date

2.1.18 SIMULATE an air flow $<200 \mathrm{cfm}$ at FIT-702K3-3B.

2.1.19 VERIFY that exhaust fan AZ702-K3-5-1B does NOT STOP.

Test Engineer Date

2.1.20 REMOVE simulation from FIT-702K3-3B .

Test Engineer Date

2.1.21 PLACE local control switch HS-AZ702K305-1B1 in the STOP position. 
2.1.22 VERIFY the following:

2.1.22.1 Exhaust Fan AZ702-K3-5-1A STOPS.

2.1.22.2 Air Conditioning Unit AZ702-K2-6-1 STOPS.

Test Engineer Date

\subsection{Fan AZ702-K3-5-1B Control from MCS}

2.2.1 SELECT Damper MK-AZ702K3-2A AUTO on MCS graphic 20BldExh.v.

2.2.2 SELECT Exhaust Fan AZ702-K3-5-1A NORMAL on MCS graphic 20BldExh.v.

2.2.3 SELECT Exhaust Fan AZ702-K3-5-1A AUTO on MCS graphic 20BIdExh.v.

2.2.4 SELECT Damper MK-AZ702K3-2B CLOSE on MCS graphic 20BIdExh.v.

2.2.5 SELECT Damper MK-AZ702K3-2B AUTO on MCS graphic 20BldExh.v.

2.2.6 PLACE the local handswitch HS-AZ702K305-1B1 in REMOTE.

2.2.7 SELECT Exhaust Fan AZ702-K3-5-1B START on MCS graphic 20BldExh.v.

2.2.8 VERIFY the following:

2.2.8.1 Damper MK-AZ702K3-2B OPENS.

2.2.8.2 Fan AZ702-K3-5-1B STARTS.

2.2.8.3 Air Conditioning Unit AZ702-K2-6-1 STARTS.

Test Engineer Date

2.2.9 After a stabilization period of 15 minutes RECORD the following data for exhaust fan AZ-K1-5-1B:

Motor Current

Motor Voltage

Amps. (II-702K35-1B)

Total Airflow

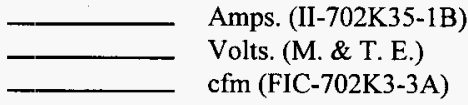

2.2.10 At the MCS SELECT HS-AZ702K305-1BB OVERRIDE command.

2.2.11 SIMULATE an air flow $<200 \mathrm{cfm}$ at FT-702K3-3B. 
PREOPERATIONAL TESTING, VENTILATION BUILDING VENTILATION SYSTEM

Revision No. 0.

WHC-SD-W030-POTP-007

Page 4 of 6.

ATTACHMENT E - VENT BUILDING TRAIN B EXHAUST FAN TEST PROCEDURE

2.2.12 VERIFY that exhaust fan AZ702-K3-5-1B does NOT STOP.

Test Engineer

Date

2.2.13 SELECT Exhaust Fan AZ702-K3-5-1B NORMAL on MCS graphic 20BldExh.v.

2.2.14 VERIFY the following:

2.2.14.1 Exhaust fan AZ702-K3-5-1B STOPS.

2.2.14.2 Damper MK-AZ702K3-2B CLOSES.

2.2.14.3 Air Conditioning Unit AZ702-K2-6-1 STOPS.

2.2.14.4 Exhaust fan AZ702-K3-5-1A STARTS.

2.2.14.5 Damper MK-AZ702K3-2A OPENS.

2.2.14.6 Air Conditioning Unit AZ702-K2-6-1 RE-STARTS.

Test Engineer

Date

2.2.15 REMOVE simulation from FIT-702K3-3B.

Test Engineer

Date

2.2.16 SELECT exhaust fan AZ702-K3-5-1B AUTO on MCS graphic 20BldExh.v.

2.2.17 OPEN circuit breaker no. 1 in Panelboard PP-16 to SIMULATE failure of exhaust fan AZ702-K3-5-1A.

2.2.18 VERIFY the following:

2.2.18.1 Exhaust fan AZ702-K3-5-1A STOPS.

2.2.18.2 Damper MK-AZ702K3-2A CLOSES.

2.2.18.3 Air Conditioning Unit AZ702-K2-6-1 STOPS.

2.2.18.4 Exhaust fan AZ702-K3-5-1B STARTS.

2.2.18.5 Damper MK-AZ702K3-2B OPENS.

2.2.18.6 Air Conditioning Unit AZ702-K2-6-1 RE-STARTS.

Test Engineer Date

2.2.19 SELECT exhaust fan AZ702-K3-5-1B STOP on MCS graphic 20BldExh.v. 
PREOPERATIONAL TESTING, VENTILATION BUILDING VENTILATION SYSTEM

Revision No. 0.

Page 5 of 6. ATTACHMENT E - VENT BUILDING TRAIN B EXHAUST FAN TEST PROCEDURE

2.2.20 VERIFY the following:

- exhaust fan AZ702-K3-5-1B STOPS.

- damper MK-AZ702K3-2B CLOSES.

Test Engineer Date 
PREOPERATIONAL TESTING, VENTILATION BUILDING VENTILATION SYSTEM

Revision No. 0.

WHC-SD-W030-POTP-007

Page 6 of 6.

ATTACHMENT E - VENT BUILDING TRAIN B EXHAUST FAN TEST PROCEDURE

\section{EXHIBIT A}

\begin{tabular}{|c|c|c|c|c|}
\hline \multicolumn{2}{|c|}{ System:Vent Bldg. Exhaust - Train A. } & Fan No.: AZ702-K3-5-1B & Fan Type: Cen & \\
\hline \multicolumn{2}{|c|}{ Drive Type: Belt. } & \multicolumn{3}{|c|}{ Service:Vent Building Ventilation } \\
\hline \multicolumn{2}{|c|}{ Equipment } & Test Data & Design & Actual \\
\hline \multicolumn{2}{|c|}{ Fan } & Total Flow (cfm) & 1000 & \\
\hline Make/Model & $\begin{array}{l}\text { American Fan } \\
\text { Co } \\
\text { RB-303-3575-24 }\end{array}$ & Disch. SP (ins. w.g.) & & \\
\hline Size & & Suct. SP (ins. w.g.) & & \\
\hline \multirow[t]{2}{*}{ Serial No. } & & Fan Total Press. (Ins. w.g.) & & \\
\hline & & Fan RPM & 3600 & \\
\hline \multicolumn{2}{|c|}{ Motor } & & & \\
\hline Make/Frame & $\begin{array}{l}\text { Westinghouse } \\
\text { /FR256T }\end{array}$ & RPM & 1755 & \\
\hline Phase & 3 & Volts & $230 / 460$ & \\
\hline SF & & FLA & $46 / 23$ & \\
\hline HP & 2 & & & \\
\hline
\end{tabular}

Vibration

\begin{tabular}{|l|l|l|l|l|l|l|}
\hline \multirow{2}{*}{ Location } & \multicolumn{2}{|c|}{ Vertical } & \multicolumn{2}{c|}{ Horizontal } & \multicolumn{2}{c|}{ Axial } \\
\cline { 2 - 7 } & Disp. (Mils) & Vel. (Ins/sec) & Disp. (Mils) & Vel. (Ins/sec) & Disp. (Mils) & Vel. (Ins/sec) \\
\hline Motor O/B & & & & & & \\
\hline Motor I/B & & & & & & \\
\hline Fan O/B & & & & & & \\
\hline Fan 1/B & & & & & & \\
\hline
\end{tabular}

Any supporting Pitot Traverse Data sheets and/or vibration spectrum printouts are to be appended to this data sheet.

ROOM/FILTER PRESSURE DIFFERENTIALS (ins. w.c)

\begin{tabular}{|l|l|l|l|l|l|l|l|}
\hline PDI-702K2-1 & & PDI-702K2-7 & & PDI-702K24-2 & & PDI-702K34-1A & \\
\hline PDIC-702K2-2 & & PDI-702K2-8 & & PDIC-702K3-1 & & PDI-702K33-1B & \\
\hline PDIC-702K2-3 & PDI-702K24-1 & & PDI-702K33- & & PDI-702K34-1B & \\
\hline
\end{tabular}




\section{ATTACHMENT F}

INITIAL CONDITIONS

1.1 VERIFY/PERFORM the electrical line-up in Attachment A, Appendix A.

Test Engineer Date

1.2 VERIFY/PERFORM the valve/damper line-up in Attachment A, Appendix B.

Test Engineer Date

1.3 OPEN manual damper MK-AZ702K3-1B.

Test Engineer Date

1.4 OPEN manual damper MK-AZ702K305-1B.

Test Engineer Date

1.5 VERIFY local control switch HS-AZ702K305-1B1 in the REMOTE position. Test Engineer Date

1.6 SET fan AZ702-K3-5-1B flow control FIC-702K3-3A $800 \mathrm{scfm}$.

1.7 SELECT Damper MK-AZ702K3-2B MANUAL on MCS graphic 20BldExh.v.

1.8 SELECT Damper MK-AZ702K3-2B OPEN on MCS graphic 20BldExh.v.

1.9 SELECT Air Conditioning Unit AZ702-K2-6-1 AUTO on MCS graphic 20BldExh.v.

1.10 SELECT exhaust fan AZ702-K3-5-1B NORMAL on MCS graphic 20BldExh.v.

1.11 SELECT exhaust fan AZ702-K3-5-1B MANUAL on MCS graphic 20BldExh.v.

\section{PROCEDURE}

2.1 START by selecting exhaust fan AZ702-K3-5-1B START command on MCS graphic 20BldExh.v.

2.2 WAIT 10 minutes for system to stabilize. 


\section{PREOPERATIONAL TESTING, VENTILATION BUILDING VENTILATION SYSTEM}

Revision No. O.

WHC-SD-W030-POTP-007

Page 2 of 2

ATTACHMENT F- VENT BUILDING TRAIN B DIFFERENTIAL PRESSURE MONITORING TEST PROCEDURE

2.3 SIMULATE an increasing pressure differential at PDE-AZ702303-1B until PDAH-AZ702303$1 \mathrm{~B}$ annunciates at the MCS.

2.4 RECORD pressure differential reading at PDI-AZ702303-1B.

PDI-AZ702303-1B Ins. w.c.(0.3 ins. w.c. expected).

Test Engineer

Date

2.5 INCREASE pressure differential simulation at PDE-AZ702303-1B until PDAHH-AZ702303-1B annunciates at the MCS.

2.6 RECORD pressure differential reading at PDI-AZ702303-1B.

PDI-AZ702303-1B Ins. w.c.(0.5 ins. w.c. expected).

Test Engineer Date

2.7 REMOVE simulation from PDE-AZ702303-1B.

Test Engineer Date

2.8 SIMULATE an increasing pressure differential at PDE-AZ702304-1B until PDAH-AZ702304IB annunciates at the MCS.

2.9 RECORD pressure differential reading at PDI-AZ702304-1B.

PDI-AZ702304-1B Ins. w.c.(3.0 ins. w.c. expected).

Test Engineer Date

2.10 INCREASE pressure differential simulation at PDE-AZ702304-1B until PDAHH-AZ702304-1B annunciates at the MCS.

2.11 RECORD pressure differential reading at PDI-AZ702304-1B.

PDI-AZ702304-1B Ins. w.c.(5.0 ins. w.c. expected).

Test Engineer Date

2.12 REMOVE simulation from PDE-AZ702304-IB.

Test Engineer Date 
PREOPERATIONAL TESTING - VENTILATION BUILDING VENTILATION SYSTEM

Revision No. 0.

WHC-SD-W030-POTP-007

Page 1 of 4.

ATTACHMENT G - VENT BUILDING AIR CONDITIONING UNIT TEST PROCEDURE

\section{ATTACHMENT G}

1 INITIAL CONDITIONS

1.1 VERIFY/PERFORM the electrical line-up detailed in Attachment A, Appendix A.

Test Engineer Date

1.2 VERIFY/PERFORM the valve/damper line-up in Attachment A, Appendix B.

Test Engineer

Date

\section{PROCEDURE}

2.1 Air Conditioning Unit AZ7(12-K2-6-1 Control from Local control switch HSAZ702K206-1A.

2.1.1 VERIFY/PLACE local handswitch HS-AZ702K206-1A in STOP position.

Test Engineer Date

2.1.2 SELECT air conditioning unit AZ702-K2-6-1 MANUAL on MCS graphic 19BldSup.v

2.1.3 SELECT air conditioning unit AZ702-K2-6-1 START on MCS graphic 19BldSup.v

2.1.4 VERIFY that air conditioning unit AZ702-K2-6-1 does NOT START.

Test Engineer Date

2.1.5 PLACE local handswitch HS-AZ702K206-1A in START position.

2.1.6 VERIFY that air conditioning unit AZ702-K2-6-1 STARTS.

Test Engineer Date 


\section{PREOPERATIONAL TESTING - VENTILATION BUILDING VENTILATION SYSTEM \\ Revision No. 0. WHC-SD-W030-POTP-007 \\ Page 2 of 4. ATTACHMENT G - VENT BUILDING AIR CONDITIONING UNIT TEST PROCEDURE}

2.1.7 SELECT air conditioning unit AZ702-K2-6-1 STOP on MCS graphic 19BldSup.v

2.1.8 VERIFY that air conditioning unit AZ702-K2-6-1 does NOT STOP.

Test Engineer Date

2.1.9 PLACE local handswitch HS-AZ702K206-1A in STOP position.

2.1.10 VERIFY that air conditioning unit AZ702-K2-6-1 STOPS.

Test Engineer Date

2.1.11 PLACE local handswitch HS-AZ702K206-1A in REMOTE position.

\subsection{Air Conditioning Unit AZ702-K2-6-1 Control from MCS}

2.2.1 SELECT air conditioning unit AZ702-K2-6-1 AUTO on MCS graphic 19BldSup.v

2.2.2 SELECT air conditioning unit AZ702-K2-6-1 START on MCS graphic 19BldSup.v

2.2.3 VERIFY that air conditioning unit AZ702-K2-6-1 does NOT START.

Test Engineer Date

2.2.4 SELECT air conditioning unit AZ702-K2-6-1 MANUAL on MCS graphic 19BldSup.v

2.2.5 SELECT air conditioning unit AZ702-K2-6-1 START on MCS graphic 19BldSup.v

2.2.6 VERIFY that air conditioning unit AZ702-K2-6-1 STARTS.

Test Engineer Date

2.2.7 SELECT air conditioning unit AZ702-K2-6-1 STOP on MCS graphic 19BldSup.v

2.2.8 VERIFY that air conditioning unit AZ702-K2-6-1 STOPS.

Test Engineer Date 
PREOPERATIONAL TESTING - VENTILATION BUILDING VENTILATION SYSTEM

Revision No. 0. WHC-SD-W030-POTP-007

Page 3 of 4. ATTACHMENT G - VENT BUILDING AIR CONDITIONING UNIT TEST PROCEDURE

2.2.9 SELECT air conditioning unit AZ702-K2-6-1 AUTO on MCS graphic 19BldSup.v

2.2.10 SELECT Damper MK-AZ702K3-2A MANUAL on MCS graphic 20BldExh.v.

2.2.11 SELECT Damper MK-AZ702K3-2A OPEN on MCS graphic 20BldExh.v.

2.2.12 SELECT exhaust fan AZ702-K3-5-1A NORMAL on MCS graphic 20BldExh.v.

2.2.13 SELECT exhaust fan AZ702-K3-5-1A MANUAL on MCS graphic 20BldExh.v.

2.2.14 SELECT exhaust fan AZ702-K3-5-1A START on MCS graphic 20BldExh.v.

2.2.15 VERIFY the following:

- exhaust fan AZ702-K3-5-1A STARTS.

- air conditioning unit AZ702-K2-6-1 STARTS.

Test Engineer Date

2.2.16 SELECT Damper MK-AZ702K3-2B MANUAL on MCS graphic 20BldExh.v.

2.2.17 SELECT Damper MK-AZ702K3-2B OPEN on MCS graphic 20BIdExh.v.

2.2.18 SELECT exhaust fan AZ702-K3-5-1B NORMAL on MCS graphic 20BldExh.v.

2.2.19 SELECT exhaust fan AZ702-K3-5-1B MANUAL on MCS graphic 20BIdExh.v.

2.2.20 SELECT exhaust fan AZ702-K3-5-1B START on MCS graphic 20BldExh.v.

2.2.21 VERIFY that exhaust fan AZ702-K3-5-1B STARTS.

2.2.22 SELECT exhaust fan AZ702-K3-5-1A STOP on MCS graphic 20BldExh.v.

2.2.23 VERIFY the following:

- $\quad$ exhaust fan AZ702-K3-5-1A STOPS.

- air conditioning unit AZ702-K2-6-1 does NOT STOP.

Test Engineer Date 
PREOPERATIONAL TESTING - VENTILATION BUILDING VENTILATION SYSTEM

Revision No. 0. $\quad$ WHC-SD-W030-POTP-007

Page 4 of 4.

ATT ACHMENT G - VENT BUILDING AIR CONDITIONING UNIT TEST PROCEDURE

2.2.24 SELECT exhaust fan AZ702-K3-5-1B STOP on MCS graphic 20BldExh.v.

2.2.25 VERIFY the following:

- $\quad$ exhaust fan AZ702-K3-5-1B STOPS.

- air conditioning unit AZ702-K2-6-1 STOPS.

Test Engineer Date 


\section{ATTACHMENT H}

\section{INITIAL CONDITIONS}

1.1 VERIFY/PERFORM the electrical line-up detailed in Attachment A, Appendix A.

Test Engineer Date

1.2 VERIFY/PERFORM the valve/damper line-up in Attachment A, Appendix B.

Test Engineer Date

1.3 VERIFY/PERFORM the following switch/control line-up:

1.3.1 SELECT Damper MK-AZ702K3-2A MANUAL on MCS graphic 20BldExh.v.

1.3.2 SELECT Damper MK-AZ702K3-2A OPEN on MCS graphic 20BldExh.v.

1.3.3 PLACE local handswitch HS-AZ702K305-1A1 in REMOTE position.

1.3.4 SELECT exhaust fan AZ702-K3-5-1A NORMAL on MCS graphic 20BldExh.v.

1.3.5 SELECT exhaust fan AZ702-K3-5-1A MANUAL on MCS graphic 20BldExh.v.

1.3.6 PLACE local handswitch HS-AZ702K206-1A in REMOTE position.

1.3.7 SELECT air conditioning unit AZ702-K2-6-1 MANUAL on MCS graphic19BldSup.v

Test Engineer

Date

1.4 At MCS graphic 20BldExh.v and 19BIdSup.v, SELECT all system Proportional-integralderivative (PID) control loops to AUTO mode; SET setpoints as follows:

$\begin{array}{llc}\text { Stack flow } & \text { FIC-702K3-3A } & 925 \mathrm{scfm} \\ & \text { FIC-702K3-3B } & 925 \mathrm{scfm} \\ \text { Vent Cell DP } & \text { PDIC-702K3-1 } & -0.75 \text { ins. wc } \\ \text { Filter room A DP } & \text { PDIC-702K2-2 } & -0.25 \text { ins. wc } \\ \text { Filter room B DP } & \text { PDIC-702K2-3 } & -0.25 \text { ins. wc }\end{array}$

1.5 During system stabilization and related data-collection periods required by this section, entire ventilation building must be isolated (unoccupied and inaccessible, with all exterior and interior doors closed). 
PREOPERATIONAL TESTING - VENTILATION BUILDING VENTILATION SYSTEM

Revision No. 0. WHC-SD-W030-POTP-007

Page 2 of 14. ATTACHMENT H - VENT BUILDING ROOM PRESSURE MONITORING AND CONTROL TEST PROCEDURE.

1.6 Test Engineer shall VERIFY the atmosphere reference leg located in the stack monitor room is sealed for use.

Test Engineer Date

\section{PROCEDURE}

NOTE: $\quad$ During testing described in this section, Test Engineer will assess system performance compared with design intent and shall describe any evaluations in the Test Log.

\subsection{Primary Vent Filter Room A Pressure Control}

2.1.1 START the building ventilation system by performing the following steps:

2.1.1.1 SELECT exhaust fan AZ702-K3-5-1A START on MCS graphic 20BldExh.v.

\subsubsection{SELECT air conditioning unit AZ702-K2-6-1 START on MCS graphic 19BIdSup.v}

2.1.2 After a stabilization period of 15 minutes RECORD the following data:

- Primary Vent Filter Room A press. diff Ins. w.c.(PDIC-AZ702K2-2)

- Valve MK-AZ702K204-1 position $\%$ Open.

NOTE: If system is deemed to operate in an unstable manner, PERFORM system PID loop tuning, step 2.8 , then restart this section.

2.1.3 SIMULATE an increase of 0.2 Ins. w.c. in Primary Vent Filter Room A pressure at PDE-AZ702K2-2. 
2.1.4 RECORD the following data:

- Primary Vent Filter Room A press. diff Ins. w.c.(PDIC-AZ702K2-2)

- Valve MK-AZ702K204-1 position $\%$ Open.

$$
\text { and VERIFY }
$$

- $\quad$ PDAL-AZ702K2-2 annunciates on MCS graphic 19BIdSup.v.

Test Engineer Date

2.1.5 ACKNOWLEDGE PDAL-AZ702K2-2 alarm on MCS graphic 19BldSup.v.

2.1.6 REMOVE simulation at PDE-AZ702K2-2.

2.1.7 RECORD the following data:

- $\quad$ Primary Vent Filter Room A press. diff

- Valve MK-AZ702K204-1 position Ins. w.c.(PDIC-AZ702K2-2) $\%$ Open. and VERIFY

- PDAL-AZ702K2-2 clears on MCS graphic 19BldSup.v.

Test Engineer Date

\subsection{Primary Vent Filter Room B Pressure Control}

2.2.1 RECORD the following data:

- Primary Vent Filter Room B press. diff Ins. w.c.(PDIC-AZ702K2-3)

- Valve MK-AZ702K204-2 position $(-0.25$ Ins. w.c. expected)

2.2.2 SIMULATE an increase of 0.2 Ins. in Primary Vent Filter Room B pressure at PDEAZ702K2-3. 
PREOPERATIONAL TESTING - VENTILATION BUILDING VENTILATION SYSTEM

Revision No. 0.

WHC-SD-W030-POTP-007

Page 4 of 14.

ATTACHMENT H - VENT BUILDING ROOM PRESSURE MONITORING AND CONTROL TEST PROCEDURE.

2.2.3 RECORD the following data:

- Primary Vent Filter Room A press. diff___ Ins. w.c.(PDIC-AZ702K2-3)

- Valve MK-AZ702K204-2 position \% Open.

and VERIFY

- PDAL-AZ702K2-3 annunciates on MCS graphic 19BldSup.v.

Test Engineer Date

2.2.4 ACKNOWLEDGE PDAL-AZ702K2-3 alarm on MCS graphic 19BldSup.v.

2.2.5 REMOVE simulation at PDE-AZ702K2-3.

2.2.6 RECORD the following data:

- Primary Vent Filter Room A press. diff___ Ins. w.c.(PDIC-AZ702K2-3)

- Valve MK-AZ702K204-2 position \% Open. and VERIFY

- $\quad$ PDAL-AZ702K2-3 clears on MCS graphic 19BldSup.v.

Test Engineer Date

\subsection{Primary Vent Cell Pressure Control}

2.3.1 RECORD the following data:

- $\quad$ Primary Vent Cell press. diff.

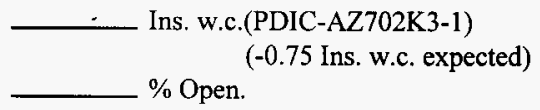

- Valve MK-AZ702K3-1 position

2.3.2 SIMULATE an increase of 0.3 Ins. w.c. in Primary Vent Cell pressure at PDEAZ702K3-1. 
PREOPERATIONAL TESTING - VENTILATION BUILDING VENTILATION SYSTEM

Revision No. 0.

2.3.3 RECORD the following data:

- Primary Vent Cell press. diff.

- Valve MK-AZ702K3-1 position Ins. w.c.(PDIC-AZ702K3-1) $\%$ Open. and VERIFY

- $\quad$ PDAL-AZ702K3-1 annunciates on MCS graphic 19BldSup.v.

Test Engineer Date

2.3.4 ACKNOWLEDGE PDAL-AZ702K3-1 alarm on MCS graphic 19BIdSup.v.

2.3.5 REMOVE simulation at PDE-AZ702K3-1.

2.3.6 RECORD the following data:

- Primary Vent Cell press. diff.

- Valve MK-AZ702K3-1 position Ins. w.c.(PDIC-AZ702K3-1) $\%$ Open. and VERIFY

- $\quad$ PDAL-AZ702K3-1 clears on MCS graphic 19BldSup.v.

Test Engineer Date

2.3.7 The following steps will test the Vent Cell Pressure Control Override feature. With system continuing to cperate as in the prior section, VERIFY Vent Cell DP with respect to atmosphere (PDIC-702K3-1 on MCS graphic 19BIdSup.v) at approximately normal DP of -0.75 ins. we.

Test Engineer Date

2.3.8 At MCS graphic 19BldSup.v, RECORD Vent Cell exhaust valve position.

Valve MK-702K3-1 $\%$ Open

Test Engineer Date 
PREOPERATIONAL TESTING - VENTILATION BUILDING VENTILATION SYSTEM

Revision No. 0. WHC-SD-W030-POTP-007

Page 6 of 14. ATTACHMENT H - VENT BUILDING ROOM PRESSURE MONITORING AND CONTROL TEST PROCEDURE.

2.3.9 In Filter Room A at PDE-702K3-2, SIMULATE a DP value more negative than the Vent Cell DP (say -1.0 ins. wc). On MCS graphic 19BldSup.v, VERIFY Vent Cell exhaust valve OPENS further to maintain a DP more negative than Filter Room A. Allow system to stabilize; RECORD values:

Valve MK-702K3-1

Vent Cell DP (PDIC-702K3-1) $\%$ Open ins. we

Test Engineer Date

2.3.10 RESTORE PDE-702K3-2 to actual value (remove simulation). VERIFY Vent Cell exhaust valve returns to normal. REPEAT above test for Filter Room B, i.e. SIMULATE - $1.0 "$ we at PDIC-702K2-3.

Test Engineer Date

\subsection{Stack Monitor Room Pressure Monitoring.}

2.4.1 RECORD the following data:

- Stack Monitor Room press. diff. Ins. w.c.(PDI-AZ702K2-1) $(-0.1$ Ins. w.c. expected)

2.4.2 SIMULATE an increase of 0.6 Ins. w.c. in Stack Monitor Room pressure at PDEAZ702K2-1.

2.4.3 RECORD the following data:

- Stack Monitor Room press. diff. Ins. w.c.(PDI-AZ702K2-1) and VERIFY

- $\quad$ PDAL-AZ702K2-1 annunciates on MCS graphic 19BldSup.v.

Test Engineer Date

2.4.4 ACKNOWLEDGE PDAL-AZ702K2-1 alarm on MCS graphic 19BldSup.v.

2.4.5 REMOVE simulation at PDE-AZ702K2-1. 
2.4.6 RECORD the following data:

- Stack Monitor Room press. diff. Ins. w.c.(PDI-AZ702K2-1) and VERIFY

- $\quad$ PDAL-AZ702K2-1 clears on MCS graphic 19BldSup.v.

Test Engineer Date

\subsection{Zone Pressure Monitoring.}

2.5.1 RECORD the following data:

- Zone I to Zone II press. diff.

- Zone II to Zone IV press. diff.

$$
\begin{gathered}
\text { Ins. w.c.(PDI-AZ702K2-7) } \\
(0.5 \text { Ins. w.c. expected) } \\
\text { Ins. w.c.(PDI-AZ702K2-8) } \\
(0.15 \text { Ins. w.c. expected) }
\end{gathered}
$$

2.5.2 DISCONNECT $1 / 4$ " tubing at low and high side connections to the following instruments:

- $\quad$ PDE-AZ702K2-2

- $\quad$ PDE-AZ702K2-3

Test Engineer Date

2.5.3 VERIFY the following:

- $\quad$ PDAH-AZ702K2-7 annunciates on MCS graphic 19BIdSup.v.

- PDAL-AZ702K2-8 annunciates on MCS graphic 19BIdSup.v.

Test Engineer Date

2.5.4 ACKNOWLEDGE PDAH-AZ702K2-7 and PDAL-AZ702K2-8 alarms on MCS graphic 19BldSup.v. 
PREOPERATIONAL TESTING - VENTILATION BUILDING VENTILATION SYSTEM

Revision No. 0.

WHC-SD-W030-POTP-007

Page 8 of 14.

ATTACHMENT H - VENT BUILDING ROOM PRESSURE MONITORING AND CONTROL TEST PROCEDURE.

2.5.5 RECONNECT $1 / 4$ " tubing at low and high side connections to the following instruments:

- PDE-AZ702K2-2

- $\quad$ PDE-AZ702K2-3

Test Engineer

Date

2.5.6 VERIFY the following:

- $\quad$ PDAH-AZ702K2-7 clears on MCS graphic 19BldSup.v.

- $\quad$ PDAL-AZ702K2-8 clears on MCS graphic 19BldSup.v.

Test Engineer

Date

2.5.7 DISCONNECT $1 / 4$ " tubing at low and high side connections to PDE-AZ702K3-1.

Test Engineer

Date

2.5.8 VERIFY that PDAL-AZ702K2-7 annunciates on MCS graphic 19BldSup.v.

Test Engineer

Date

2.5.9 ACKNOWLEDGE PDAL-AZ702K2-7 alarm on MCS graphic 19BldSup.v.

2.5.10 RECONNECT $1 / 4$ " tubing at low and high side connections to PDE-AZ702K3-1.

Test Engineer

Date

2.5.11 VERIFY that PDAL-AZ702K2-7 clears on MCS graphic 19BldSup.v.

Test Engineer

Date

2.5.12 DISCONNECT $1 / 4$ " tubing at low and high side connections to PDE-AZ702K2-1.

Test Engineer Date

2.5.13 VERIFY that PDAH-AZ702K2-8 annunciates on MCS graphic 19BldSup.v.

Test Engineer Date

2.5.14 ACKNOWLEDGE PDAH-AZ702K2-8 alarm on MCS graphic 19BldSup.v. 
PREOPERATIONAL TESTING - VENTILATION BUILDING VENTILATION SYSTEM

Revision No. 0.

WHC-SD-W030-POTP-007

Page 9 of 14.

ATTACHMENT H - VENT BUILDING ROOM PRESSURE MONITORING AND CONTROL TEST PROCEDURE.

2.5.15 RECONNECT 1/4" tubing at low and high side connections to PDE-AZ702K2-1.

Test Engineer

Date

2.5.16 VERIFY that PDAH-AZ702K2-8 clears on MCS graphic 19BldSup.v.

Test Engineer

Date

\subsection{Emergency Cooling Valves Function}

2.6.1 On MCS graphic 22BldTmp.v, RECORD the following data:

$\begin{array}{lll}0 & \text { Room temperature (E\&I-A) } & \text { F (TI-702K2-2) } \\ \circ & \text { Room temperature (E\&I-B) } & \text { F (TI-702K2-1) } \\ 0 & \text { Room temperature (E\&I-A) } & \text { F (TI-702K2-3) }\end{array}$

(expected range $60-\overline{85} \mathrm{~F}$ )

2.6.2 At MCS graphic 22BldTmp.v, VERIFY the following HSs and MOVs CLOSED:

o HS-AZ702K2-7, MK-AZ702-K2-7A, -7B

$0 \quad$ HS-AZ702K2-6, MK-AZ702-K2-6A, -6B

$0 \quad$ HS-AZ702K2-8, MK-AZ702-K2-8A, -8B

Test Engineer

Date

2.6.3 At MCS graphic 22BldExh.v, SELECT remote handswitch HS-AZ702K2-7 OPEN.

2.6.4 VERIFY valves MK-AZ702K2-7A, -7B OPEN within 20 seconds.

Test Engineer Date

2.6.5 REPEAT prior two steps. VERIFY repeatability.

Test Engineer Date

2.6.6 At MCS graphic 22BIdTmp.v, SELECT remote handswitch HS-AZ702K2-6 OPEN.

2.6.7 VERIFY valves MK-AZ702K2-6A, -6B OPEN within 20 seconds.

Test Engineer Date

2.6.8 REPEAT prior two steps. VERIFY repeatability.

Test Engineer Date 
PREOPERATIONAL TESTING - VENTILATION BUILDING VENTILATION SYSTEM

Revision No. 0.

WHC-SD-W030-POTP-007

Page 10 of 14.

ATTACHMENT H - VENT BUILDING ROOM PRESSURE MONITORING AND CONTROL TEST PROCEDURE.

2.6.9 At MCS graphic 22BldTmp.v, SELECT remote handswitch HS-AZ702K2-8 OPEN.

2.6.10 VERIFY valves MK-AZ702K2-8A, -8B OPEN within 20 seconds.

Test Engineer Date

2.6.11 REPEAT prior two steps. VERIFY repeatability.

Test Engineer Date

2.6.12 At MCS graphic 22BldTmp.v, SELECT remote handswitches HS-AZ702K2-6, -7, -8 NORMAL. VERIFY all six related valves closed.

Test Engineer Date

2.6.13 At TT-AZ702K2-2 IN E\&I ROOM-A, SIMULATE a temperature increase until TAHH alarm occurs on MCS graphic 22BldTmp.v.

2.6.14 VERIFY valve MK-AZ702K2-7A OPEN on MCS graphic 22BldTmp.v.

2.6.15 For prior step, RECORD temperature indication at TAHH alarm point: TI-AZ702K2-2 F (expected, 110 F)

2.6.16 At TT-AZ702K2-1 IN E\&I ROOM-A, SIMULATE a temperature increase until TAHH alarm occurs on MCS graphic 22BldTmp.v.

2.6.17 VERIFY valve MK-AZ702K2-6A OPEN on MCS graphic 22BldTmp.v.

2.6.18 For prior step, RECORD temperature indication at TAHH alarm point: TI-AZ702K2-1 F (expected, $110 \mathrm{~F})$

2.6.19 At TT-AZ702K2-3 IN E\&I ROOM-A, SIMULATE a temperature increase until TAHH alarm occurs on MCS graphic 22BldTmp.v.

2.6.20 VERIFY valve MK-AZ702K2-8A OPEN on MCS graphic 22BIdTmp.v.

2.6.21 For prior step, RECORD temperature indication at TAHH alarm point: TI-AZ702K2-3 F (expected, $110 \mathrm{~F})$ 


\subsection{Vent Building Vent System MCS MACRO Test}

2.7.1 PLACE system in automatic mode as follows: At MCS graphic 20BldExh.v, SELECT all components to NORMAL.

2.7.2 At MCS graphic 27mBld.v, SELECT AC "ON", and Fan 1A "ON". RUN MACRO, VERIFY system STARTS with AC and Fan $1 A$ ON.

Test Engineer Date

2.7.3 At MCS graphic 27mBld.v, SELECT AC and Fan $1 B$ "ON". RUN MACRO and VERIFY AC and Fan $1 B$ are ON, Fan 1A OFF.

Test Engineer Date

2.7.4 At MCS graphic 27mBld.v, SELECT AC OFF, Fan 1B ON. RUN MACRO. VERIFY $A C$ is OFF and Fan $1 B$ stays $O N$.

Test Engineer Date

\subsection{Final Tuning of System PID Controls by MCS Cognizant Engineer}

2.8.1 At MCS graphic 20BldExh.v and 19BldSup.v, SELECT all PID controls in AUTO mode. At MCS graphic 27mBld.v, SELECT AC "ON", and Fan 1 A "ON". RUN MACRO, VERIFY system STARTS with AC and Fan $1 B$ ON. RUN system for 10 minutes to stabilize; MAINTAIN Vent Bldg. in isolation mode during this entire section.

Test Engineer Date

2.8.2 At MCS graphic 20BldExh.v and 19BldSup.v, RECORD the following data:

Stack flow

FIC-702K 3-3A

FIC-702K3-3B

Vent Cell DP

PDIC-702K $3-1$

Filter room A DP

PDIC-702K2-2

Filter room B DP

PDIC-702K2-3

Stack Mon. room DP

PDI-702K2-1

Zone I/II DP

PDI-702K2-7

Zone II/IV DP

PDI-702K2-8

scfm scfm ins. we ins. we ins. wc

ins. wc ins. wc ins. wc 
PREOPERATIONAL TESTING - VENTILATION BUILDING VENTILATION SYSTEM

Revision No. 0.

WHC-SD-W030-PO'TP-007

Page 12 of 14.

ATTACHMENT H - VENT BUILDING ROOM PRESSURE MONITORING AND CONTROL TEST PROCEDURE.

2.8.3 At MCS Engineering station, TUNE PID loops as follows. RECORD initial PID rate settings for above controllers in the table below. Then REPEAT for Train B.

ADJUST the Proportional, Integral and Derivative control gains for stable control of each loop. This will first be performed on each individual loop, and may have to be repeated when the loops operate interactively. Use an empirical technique and include the following steps:

- Increase the loop gain, forcing the system to oscillate, and determine the maximum gain and oscillation frequency.

- $\quad$ Adjust the P, I, D rates for desired system performance and test various transients for stability and desired operation.

\begin{tabular}{||l|c|c||}
\hline \multicolumn{1}{|c|}{ PARAMETER } & \multicolumn{2}{|c|}{ INITIAL PID RATES } \\
\cline { 2 - 4 } & Train A & Train B \\
\hline Stack flow, FIC-702K3-3A & & \\
\hline Stack flow, FIC-702K3-3B & & \\
\hline Vent Cell diff press, PDIC-702K3-1 & & \\
\hline $\begin{array}{l}\text { Filter Room-A diff press, PDIC- } \\
\text { 702K2-2 }\end{array}$ & & \\
\hline $\begin{array}{l}\text { Filter Room-B diff press, PDIC- } \\
\text { 702K2-3 }\end{array}$ & & \\
\hline
\end{tabular}

2.8.4- PERFORM the upset actions described below in order to determine system response to each. RECORD observations, corresponding PID adjustment settings, and final system response observations; PERFORM PID tuning according to the following criteria.

- Stack flow- Maintain indicated stack áirflow at $925+/-50 \mathrm{sefm}$, under steadystate conditions; during defined upset conditions (as described below), flow shall remain between the high and low alarm levels. Control should be tuned for a highly stable flow signal.

- Vent Cell pressure- Maintain indicated Vent Cell DP (with respect to atmosphere) at $-0.75+/-0.1$ ins. wc under steady-state conditions; normal DP shall be automatically restored following each defined upset condition such that no alarm occurs, during or following the upset. Override control shall activate within 15 seconds after abnormal DP occurs, and restore safe and stable DP within another 15 seconds (i.e. Zone I/II DP negative). 


\section{PREOPERATIONAL TESTING - VENTILATION BUILDING VENTILATION SYSTEM \\ Revision No. 0.- $\quad$ WHC-SD-W030-POTP-007 Page 13 of 14. ATTACHMENT H - VENT BUILDING ROOM PRESSURE MONITORING AND CONTROL TEST PROCEDURE.}

- Filter Room pressure- Maintain indicated Filter Room DP at $-0.25+/-0.05$ ins. wc (to atmosphere) under steady-state conditions; normal DP shall be automatically restored following each defined upset condition, such that no alarm occurs, during or following the upset. Also, no Zone-II/IV alarm shall occur.

- Stack Monitor Room pressure- Maintain indicated room DP at $-0.1+/-0.05$ ins. wc (to atmosphere) under steady-state conditions; normal DP shall be automatically restored following each defined upset condition such that no alarm occurs, during or following the upset. Also, no Zone-II/IV alarm shall occur.

2.8.5 RECORD data for Upset-1; STOP AC unit; RE-START after 5 minutes:

2.8.6 RECORD data for Upset-2; HOLD open outer door to E\&I room A:

2.8.7 RECORD data for Upset-3; HOLD open outer door to E\&I room B:

2.8.8 RECORD data for Upset-4; OPEN interior door between E\&I room A and Stack Monitor room (hold 10 seconds):

2.8.9 RECORD data for Upset-5; BRIEFLY OPEN both outer doors to Fan Room A (hold 10 seconds): 
2.8.10 RECORD data for Upset-6; BRIEFLY OPEN both outer doors to Fan Room B (hold 10 seconds):

2.8.11 RECORD data for Upset-7; SWITCH exhaust fans:

2.8.12 VERIFY that all PID control adjustments are complete.

MCS Cog. Engineer

Test Engineer

Date Date

\subsection{Alarm Logic Timing Checks by MCS Cognizant Engineer}

2.9.1 At MCS graphic 20BIdExh.v and 19BldSup.v, SELECT all PID controls in AUTO mode, Fan $1 A$ to STOP. At MCS graphic 27mBld.v, SELECT AC "OFF", and Fan $1 B$ "ON". RUN MACRO, verify system STARTS with AC OFF and Fan 1B ON. RUN system for 10 minutes to stabilize; maintain Vent Bldg. in isolation mode during this entire section. PREPARE MCS alarm printer to print alarms as noted below.

Test Engineer Date

2.9.2- At MCS graphic 20EldExh.v, SELECT Fan 1B to STOP. RECORD alarm level and time required for each of the following alarms to occur after fan STOP command.

Stack flow FAL-FIC-702K3-3A
FAL-FIC-702K3-3B
PDAL-PDIC-702K3-1
PDAL-PDIC-702K2-2
PDAL-PDIC-702K2-3
DPPDAL-PDI-702K2-1
PDAL-PDI-702K2-7
PDAL-PDI-702K2-8

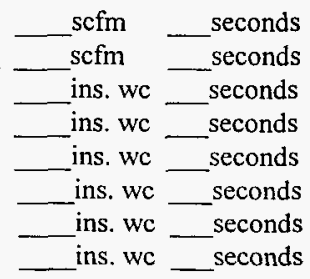

2.9.3 VERIFY that all alarm logic timing is satisfactory.

MCS Cog. Engineer Test Engineer Date Date 
PREOPERATIONAL TESTING - VENTILATION BUILDING VENTILATION SYSTEM

Revision No. 0 WHC-SD-W030-POTP-007

PAGE 1 OF 39

ATTACHMENT I - VENT BUILDING VENTILATION STACK MONITOR TEST PROCEDURE

1.0 INITIAL CONDITIONS

1.1 Vent and Balance group are available to support testing.

Test Engineer:

2.0 VERIFY all system instrumentation in Appendix A is calibrated and has a current calibration tag affixed to each instrument and that all system loop calibrations are complete.

Test Engineer:

3.0 VERIFY the SYSTEM IS ALIGNED for preoperational testing in accordance with Appendix B.

Test Engineer:

4.0 CYCLE all MANUAL VALVES, listed in Appendix C, full travel and verify proper operation.

Test Engineer:

4.1 RECORD ALL Valve Deficiencies in Attachment K, Test Log.

\subsection{VENTILATION BUILDING VENTILATION STACK MONITOR}

5.1 VERIFY that system has been energized for a minimum of one hour prior to recording data in in step 5.29.

Test Engineer:

5.2 VERIFY flow and sample probe assembly is correctly oriented and securely mounted in place.

Test Engineer.:

5.3 VERIFY instrumentation cabinet is securely mounted to floor/pad.

Test Engineer:

5.4 VERIFY all signal connections between flow and sample probe and instrumentation/control cabinet are correct. These include Record sample transport line, Beta sample transport line, total and static pressure signal lines, stack temperature signal, power wiring, and signal wiring.

Test Engineer.:

5.5 VERIFY sample exhaust from cabinet is routed back to the stack.

Test Engineer: 
5.6 VERIFY incoming electrical power is connected to the terminal blocks TB-1 and TB-2.

Test Engineer:

5.7 VERIFY all wiring for remote signals is properly connected to terminal block TB-6.

Test Engineer.:

5.8 VERIFY stack exhaust fan status wiring is connected to terminal block TB-4.

Test Engineer.:

5.9 VERIFY PUMP AUTO/OFF selector switches AZ702K3-11-1 and AZ702K3-11-2 are in the OFF position.

Test Engineer:

5.10 PLACE Power Switch SW-1 in the ON position.

5.11 VERIFY POWER ON light $L-1$ is ON.

5.12 VERIFY sample cabinet heater fan starts.

Test Engineer.:

Test Engineer.:

5.13 PLACE Power Switch SW-2 in the ON position.

5.14 VERIFY instrumentation cabinet cooling fan starts.

Test Engineer.:

5.15 ENERGIZE the Power Module.

5.16 VERIFY displays in FT-1, FT-2, and FT-3 are ON.

Test Engineer.:

5.17 Place Power Switch SW-3 in the ON position.

5.18 VERIFY display in Beta monitor is ON.

Test Engineer.: 
5.19 DEPRESS alarm acknowledge button PB-1 AND VERIFY the light in the button comes ON. Test Engineer.:

5.20 VERIFY that a building exhaust fan is running.

Test Engineer.:

5.21 PLACE Sample Pump AZ702-K3-11-1 local control switch HS-AZ702K311-1 in AUTO.

5.22 VERIFY Sample Pump AZ702-K3-11-1 starts.

Test Engineer.:

5.23 PLACE Sample Pump AZ702-K3-11-2 local control switch HS-AZ702K311-2 in AUTO.

5.24 VERIFY Sample Pump AZ702-K3-11-2 starts.

Test Engineer.:

5.25 STOP the building exhaust fan by placing it's control switch in OFF on graphics 20 bldExh.v.

5.26 VERIFY Sample Pumps AZ702-K3-11-1 and AZ702-K3-11-2 stop.

Test Engineer.:

5.27 PLACE Sample Pump local control switches HS-AZ702K311-1 and HS-AZ702K311-2 in OFF.

5.28 DEPRESS alarm acknowledge but PB-1 AND VERIFY the light in the button goes OFF.

Test Engineer.:

5.29 Sample Flow Control Loop

NOTE - $\quad$ Record required data for this section on Appendix D

5.29.1 START a building ventilation exhaust fan from graphics screen 20BIdExh.v.

5.29.2 PLACE Sample Pump local control switches HS-AZ702K311-1 and HSAZ702K $311-2$ in auto.

5.29.3 VERIFY Sample Pumps AZ702-K3-11-1 and AZ702-K3-11-2 start.

Test Engineer: 
5.29.4 ADJUST Ventilation Stack Flow to approximately 960 SCFM using the stack flow controller for the operating exhaust fan on graphics screen 20BldExh.v.

5.29.5 CALCULATE percent of full scall (\%FS) stack flow using stack flow (SCFM) displayed on FI-AZ702K3-3 and the following formula:

NOTE - $\quad 1050$ SCFM represents full scale stack flow.

$$
\% \text { FS Stack Flow }=(\text { Displayed SCFM } \div 1050 \text { SCFM } X 100
$$

5.29.6 RECORD the value for displayed stack flow and the \%FS Stack Flow calculated in step 5.29.5.

Test Engineer.:

5.29.7 CALCULATE Ideal Output of V/I-1 channel 1 using \%FS calculated in step 5.29.5 and the following formula:

$$
\text { Ideal Output }=(\% \text { SS Stack Flow } \div 100) \times 16.00 \mathrm{~mA}+4 \mathrm{~mA}
$$

5.29.8 USING a DMM, MEASURE AND RECORD the output of V/I -1 channel 1 .

Test Engineer.:

5.29.9 CALCULATE the percent deviation from the ideal output, caluclated in step 5.29.7. This deviation should be $\leq 2 \%$.

$\%$ Deviation $=[$ Actual Output $(m A)-4 m A]-[$ deal Output $(m A)-4 m A]$

Ideal Output $(m A)-4 m A$

5.29.10 RECORD \%Deviation calculated in step 5.29.9.

Test Engineer.:

5.29.11 CALCULATE Ideal Sample Flow for Record and Beta Samples using \%FS Stack Flow calculated in step 5.29.5 and the following formula:

NOTE - $\quad 2$ SCFM represents full scale sample flow.

Ideal Sample Flow $=\%$ FS Stack Flow $\div 100 \times 2.00$ SCFM 
5.29.12 RECORD the Ideal Sample Flow calculated in step 5.29.11.

Test Engineer:

5.29.13 RECORD sample flows as indicated on FIC-AZ702K3-1 and FIC-AZ702K32.

Test Engineer.:

5.29.14 CALCULATE the \% Deviation, for the Record sample flow, from the Ideal Sample flow calculated in step 5.29.11. This deviation should be $\leq 5 \%$.

Record Sample Flow \% Deviation = Displayed SCFM - Ideal SCFMX 100

Ideal SCFM

5.29.15 RECORD the Record Sample Flow \% Deviation calculated in step 5.29.14.

Test Engineer.:

5.29.16 CALCULATE the \% Deviation, for the Beta sample flow, from the Ideal Sample flow calculated in step 5.29.11. This deviation should be $\leq 5 \%$.

Beta Sample Flow \% Deviation $=$ Displaved SCFM-Ideal SCFM X 100

Ideal SCFM

5.29.17 RECORD Beta Sample Flow \% Deviations calculated in step 5.29.16.

Test Engineer.:

5.29.18 CALCULATE the ideal output for V/I-1 channel 2 using the displayed sample flow recorded in step 5.29.13.

Channel 2 Ideal Output $=\underline{\text { Displaved SCFM }} \times 16.00 \mathrm{~mA}+4.00 \mathrm{~mA}$ 2.00 SCFM

5.29.19 RECORD the Channel 2 Ideal Output calculated 5.29.18.

Test Engineer.:

5.29.20 CALCULATE the ideal output for V/I-1 channel 3 using the displayed sample flow recorded in step 5.29.13.

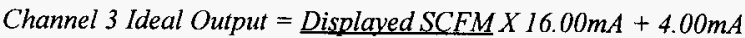


PREOPERATIONAL TESTING - VENTILATION BUILDING VENTILATION SYSTEM

5.29.21 RECORD the Channel 3 Ideal Output calculated 5.29.20.

Test Engineer.:

5.29.22 USING a DMM, MEASURE AND RECORD the output of V/I - 1 channel 2 and 3 .

5.29.23 CALCULATE the \% Deviation from the ideal output for the Record sample . flow. This deviation should be $\leq 2 \%$.

$\%$ Deviation $=[$ Actual Output $(m A)-4 m A]-[$ deal Output $(m A)-4 m A]$

$$
\text { Ideal Output (mA) - } 4 \mathrm{~mA}
$$

5.29.24 RECORD \% Deviation, for the Record sample flow, from ideal output calculated 5.29.23.

Test Engineer.:

5.29.25 CALCULATE the \% Deviation from the ideal output for the Beta sample flow. This deviation should be $\leq 2 \%$.

$\%$ Deviation $=[$ Actual Output $(m A)-4 m A]-[$ deal Output $(m A)-4 m A]$

Ideal Output (mA) - $4 \mathrm{~mA}$

5.29.26 RECORD \% Deviation, for the Beta sample flow, from ideal output calculated in step 5.29.25.

Test Engineer.:

5.29.27 CALCULATE the percent full scale (\%FS) stack temperature using stack temperature indicated on TI-AZ702K3-1.

NOTE - $\quad 150^{\circ} \mathrm{F}$ represents full scale stack temperature.

$\%$ FS Stack Temperature $=\frac{\text { Displayed }^{\circ} \mathrm{F}}{150^{\circ} \mathrm{F}} 100$

5.29.28 RECORD Full stack temperature and \%FS Stack Temperature calculated in step 5.29.27.

Test Engineer.: 


\section{PREOPERATIONAL TESTING - VENTILATION BUILDING VENTILATION SYSTEM}

5.29.29 CALCULATE the ideal output of V/I -2 channel 2 using the \%FS Stack Temperature calculated in step 5.29.27.

$$
\text { Ideal Output }(\mathrm{mA})=\% \text { FS Stack Temperature } X 16.00 \mathrm{~mA}+4 \mathrm{~mA}
$$

$$
100
$$

5.29.30 RECORD V/l channel 2 Ideal Output calculated in step 5.29.29.

Test Engineer.:

5.29.31 USING a DMM, MEASURE AND RECORD the output of V/I -2 channel 2.

Test Engineer.:

5.29.32 CALCULATE the \% Deviation, for V/I -2 channel 2, from the Ideal output calculated in step 5.29.29. This deviation should be $\leq 2 \%$.

$\%$ Deviation $=[$ Actual Output $(m A)-4 m A]-[\operatorname{ldeal}$ Output $(m A)-4 m A]$

Ideal Output ( $\mathrm{mA})$ - $4 \mathrm{~mA}$

5.29.33 RECORD the \% Deviation for V/I -2 channel 2 calculated in step 5.29.32.

Test Engineer.:

5.29.34 REMOVE all DMMs and reconnect all wiring.

Test Engineer:

5.30 Beta Monitor

NOTE - Record all data from this section on Appendix E.

5.30.1 INSTALL a known check source into remote detector head and allow sufficient time for monitor to perform count.

5.30.2 CALCULATE the ideal output for RE-AZK 1-1 using the displayed value. $3.77^{\wedge}-4$ DPS represents fullscale value.

Ideal Output $(\mathrm{mA})=$ Displayed Value $X 16.00 \mathrm{~mA}+4 \mathrm{~mA}$

$3.77^{\wedge}-4$ DPS (Full Scale) 
5.30.3 RECORD the ideal output calculated in step 5.30.2.

Test Engineer.:

5.30.4 USING a DMM, MEASURE and RECORD the current output of RE-AZK1-1.

Test Engineer.:

5.30.5 CALCULATE the percent deviation from the ideal output calculated in step 5.30.2.

$\%$ Deviation $=[$ Actual Output $(m A)-4 m A]-[$ deal Output $(m A)-4 m A\}$

Ideal Output (mA) - $4 \mathrm{~mA}$

5.30.6 RECORD the actual output and the percent deviation calculated in step 5.30.5.

Test Engineer.:

5.30.7 REPEAT steps 5.30.1 through 5.30 .6 for all check sources to be used.

5.30.8 REMOVE DMM and reconnect all wiring.

5.30.9 CREATE a failure of the Beta Monitor by installing a jumper from $41 \mathrm{RD}$ to $\mathrm{H} 1 \mathrm{RD}$ on the back of the Beta Monitor.

5.30.10 VERIFY the following events occur:

- The local horn sounds.

- The local amber beacon flashes (both are on top of the instrumentation cabinet).

Test Engineer.:

5.30.11 DEPRESS the horn acknowledge button PB-1.

5.30.12 VERIFY the following events occur:

- The horn stops sounding.

- The amber beacon continues to flash.

- The light in PB-1 is ON.

Test Engineer.:

5.30.13 REMOVE the jumper installed in Step 5.30.10 
PREOPERATIONAL TESTING - VENTILATION BUILDING VENTILATION SYSTEM

5.30.14 VERIFY the amber beacon stops flashing.

Test Engineer.:

5.30.15 DEPRESS acknowledge button PB-1.

5.30.16 VERIFY hom does not sound and light in PB-1 is OFF.

Test Engineer.:

5.30.17 CREATE a high radiation condition of the Beta Monitor by installing a jumper from terminal $40 \mathrm{RD}$ to $\mathrm{H} 1 \mathrm{RD}$ on the back of the Beta Monitor.

5.30.18 VERIFY the horn sounds and the local red beacon flashes.

Test Engineer.:

5.30.19 DEPRESS the horn acknowledge button PB-1.

5.30.20 VERIFY the following events occur:

- The horn stops sounding.

- The red beacon continues to flash.

- The light in PB-1 is ON.

Test Engineer.:

5.30.21 REMOVE the jumper installed in Step 5.30.17.

5.30.22 VERIFY the red beacon stops flashing.

Test Engineer.:

5.30.23 DEPRESS horn acknowledge button PB-1.

5.30.24 VERIFY horn does not sound and light in PB-1 is OFF.

Test Engineer.: 
5.31 Test for Accuracy, Zero Drift and Calibration Drift.

5.32 SELECT Damper MK-AZ702K3-2A CLOSE on MCS graphic 20BldExh.v.

5.33 SELECT Damper MK-AZ702K3-2A AUTO on MCS graphic 20BldExh.v.

5.34 PLACE the local handswitch HS-AZ702K305-1A1 in REMOTE.

5.35 At the MCS SELECT HS-702K35-1A START command.

5.36 VERIFY the following:

- damper MK-AZ702K3-2A OPENS.

- fan AZ702-K3-5-1A STARTS.

5.36.1 INSTALL the Reference Method Apparatus.

NOTE - The Stack monitoring system will be operated for a 24 hour conditioning period, during which it shall be continuously measuring stack flow.

5.36.2 RECORD the start date and time for this portion of the test.

Start Date

Time

Test Engineer:

5.36.3 DURING the 24 hour test, take three sets of flow measurements at least one hour apart, using the reference method and the monitoring system.

NOTE: Additional data sheets will be provided for this portion of the test.

5.36.4 COLLECT Reference Method date using 6-TF-155, Air Flow Test for Tank Farm Stacks and Ducts.

5.36.5 RECORD reference method data taken in step 5.36.3 on Appendix H.

Test Engineer:

5.36.6 RECORD data taken in step 5.36.3 on Appendix F.

Test Engineer 
5.36.7 DURING the 24 hour test, subject the monitoring system to the manufacturers' specified zero and calibration procedures, after the first set of flow data and after the second set of flow data, to test for calibration drift and zero drift.

5.36.8 RECORD the monitoring system output reading (Volts DC) on Appendix G before and after the adjustments in step 5.36.7.

Test Engineer.:

5.37 UPON completion of steps 5.36.1 through 5.36.8 THEN PERFORM the following calculations on Appendix F:

5.37.17 CALCULATE the mean (average) value of the 3 reference method values (volumetric) using the following equation:

$\overline{R M}$ (mean reference method value) $=\sum_{1}^{2} R M \div 3$

where, $\sum R M=$ sum of the individual reference method values.

5.37.2 CALCULATE the individual differences between the 3 pairs of reference method values $(R M)$ and the measurement system values $(M S)$ using the following equation:

$$
d=R M-M S
$$

5.37.3 CALCULATE the sum of the differences of the 3 pairs calculated in step 5.37.2.

$$
\dot{\sum} d
$$

5.37.4 CALCULATE the mean value of the differences of the 3 pairs caluclated above, using the following equation:

$$
\bar{d} \text { (mean difference })=\sum_{1} d \div 3
$$

5.37.5 CALCULATE the square of each of the 3 values of calculated in step 5.37.2. 
5.37.6 CALCULATE the sum of the squares of the differences calculated in step 5.37.5.

$$
\dot{\sum} d
$$

5.37.7 PERFORM the following calculations for both Zero and Calibration on Appendix G:

5.37.8 CALCULATE the individual differences between the 2 pairs of successive readings.

5.37.9 CALCULATE the sum of the differences of the 2 pairs calculated in step 5.37.8.

$$
\sum^{2} d
$$

5.37.10 CALCULATE the mean value of the differences for the 2 pairs, using the following equation:

$$
\bar{d}=\sum_{1}^{2} d_{1} \div 2
$$

5.37.11 CALCULATE the square of each of the 2 values of 5.37.8.

5.37.12 CALCULATE the sum of the squares of the differences calculated in step 5.37.11.

$$
\dot{\sum} d
$$


CALCULATE the Relative Accuracy of the measurement system versus the reference method used in the following equation:

$$
R A=\frac{|d| \div c c}{\overline{R M}} \times 100
$$

$\overline{R M}=$ ari thmatic mean of the reference method.

$\bar{d}=$ the absolute value of the mean difference between the reference method values (volumetric) and the corresponding measurement system values (volumetric) $\mathrm{cc}=$ confidence coefficient

$$
\text { where } c c=t_{\text {osst }} \frac{S d}{\sqrt{3}}
$$

$$
T_{0025} \text { for } 3=4.303
$$

$$
S d=\sqrt{\sum_{1}^{3} d^{2}-\left[\frac{\left(\sum_{1}^{\infty} d\right)^{2}}{3}\right]}
$$

NOTE - $\quad$ The values of $\overline{R M}, \bar{d}, \sum_{1} d_{1}^{2}$, and $\sum_{1}^{2} d_{1}$ can be found on Appendix F.

5.37.14 RECORD the values calculated for Sd, cc, and RA in Appendix F.

Test Engineer.: 
CALCULATE the Zero Drift of the measurement system using the following equation:

$$
\text { Zero Drift }=\left[\frac{|\bar{d}| \div c c}{5 \mathrm{~V}}\right] \times 100
$$

where,

$d=$ the absolute value of the mean difference between the 2 pairs of successive readings. $\mathrm{cc}=$ confidence coefficient

$$
\text { Where } c c=t_{\text {ons }} \frac{S d}{\sqrt{2}}
$$

$$
\mathrm{T}_{0.025} \text { for } 2=12.706
$$

$$
S d=\sqrt{\sum_{1}^{2} d^{2}-\left[\frac{\left(\sum_{1}^{2} d\right)^{2}}{2}\right]}
$$

NOTE - The values of $\bar{d}, \sum_{1}^{2} d_{1}^{2}$, and $\sum_{1}^{2} d$ can be found on Appendix G.

5.37.16 RECORD the values calculated for Sd, cc, and Zero Drift on Appendix G. 
CALCULATE the Calibration Drift of the measurement system using the following equation:

$$
\text { Calibration Drift }=\left[\frac{|\bar{d}| \div c c}{5 V}\right] \times 100
$$

where,
$|d|=$ the absolute value of the mean difference between the 2 pairs of successive readings.
$\mathrm{cc}=\quad$ confidence coefficient

$$
\text { Where } c c=t_{\operatorname{sou}} \frac{S d}{\sqrt{2}}
$$

$$
\mathrm{T}_{0.025} \text { for } 2=12.706
$$

$$
S d=\sqrt{\sum_{1}^{2} d_{1}^{2}-\left[\frac{\left(\sum_{1}^{2} d\right)^{2}}{2}\right]}
$$

NOTE - The values of $\bar{d}, \sum_{1}^{2} d_{1}^{2}$, andc $\sum_{1}^{2} d_{1}$ an be found on Appendix G.

5.37.18 RECORD the values calculated for Sd, cc, and Calibration Drift on Appendix G. 
PREOPERATIONAL TESTING - VENTILATION BUILDING VENTILATION SYSTEM

Revision No. 0 WHC-SD-W030-POTP-007

PAGE 16 OF 39

ATTACHMENT I - VENT BUILDING VENTILATION STACK MONITOR TEST PROCEDURE

5.37.19 ACCEPTANCE CRITERIA for Relative Accuracy, Zero Drift, and Calibration Drift are as follows;

- Relative Accuracy $\quad<10 \%$ of $\overline{R M}$

- Zero Drift $\quad<3 \%$ of Span

- Calibration Drift $<3 \%$ of Span

5.38 Stack Monitor Roof Cabinet Cooling Fan

5.38.1 VERIFY that detector head in cabinet is operating.

Test Engineer

Date

5.38.2 VERIFY that internal recirculation fan is OFF when cabinet

temperature is below $80 \mathrm{~F}$.

Test Engineer

Date

5.38.3 VERIFY that internal recirculation fan is ON when cabinet temperature is above $100 \mathrm{~F}$.

Test Engineer

Date

5.38.4 VERIFY that internal cabinet temperature is a maximum of $5 \mathrm{~F}$ warmer than ambient under the following conditions: Ambient temperature is above $100 \mathrm{~F}$, bright sun fully directed on cabinet for a minimum of 2 hours. RECORD actual conditions.

Ambient outdoor temperature:

Equilibrium temperature inside cabinet: ——F Test Engineer

Date

\section{SECURE FROM POTP}

6.1 VERIFY that all temporary test equipment has been removed from the Ventilation Building Ventilation System.

Test Engineer:

6.2 VERIFY that all temporary test equipment has been removed from the Ventilation Building Ventilation Stack Monitor.

Test Engineer: 
PREOPERATIONAL TESTING - VENTILATION BUILDING VENTILATION SYSTEM

Revision No. 0

ATTACHMENT I - VENT BUILDING VENTILATION STACK MONITOR TEST PROCEDURE

\begin{tabular}{|c|c|c|c|}
\hline Equipment Number & Functional Description & Signature & Date/Time \\
\hline FT-AZ702K3-1 & $\begin{array}{l}\text { Beta Monitor Sampler Flow } \\
\text { Transmitter in Monitor Panel in } \\
\text { Stack Monitor Room. }\end{array}$ & & \\
\hline FT-AZ702K3-2 & $\begin{array}{l}\text { Record Monitor Sampler Flow } \\
\text { Transmitter in Monitor Panel in } \\
\text { Stack Monitor Room. }\end{array}$ & & \\
\hline FT-AZ702K3-3A & $\begin{array}{l}\text { Stack Discharge Flow } \\
\text { Transmitter in Monitor Panel in } \\
\text { Stack Monitor Room. }\end{array}$ & & \\
\hline FT-AZ702K3-3B & $\begin{array}{l}\text { Stack Discharge Flow } \\
\text { Transmitter in Monitor Panel in } \\
\text { Stack Monitor Room. }\end{array}$ & & \\
\hline PI-AZ702K3-1 & $\begin{array}{l}\text { Beta Monitor Sampler Pressure } \\
\text { Gauge in Monitor Panell in Stack } \\
\text { Monitor Room. }\end{array}$ & & \\
\hline PI-AZ702K3-2 & $\begin{array}{l}\text { Record Monitor Sampler } \\
\text { Pressure Gauge in Monitor Panel } \\
\text { in Stack Monitor Room. }\end{array}$ & & \\
\hline RI-AZ702K3-1 & $\begin{array}{l}\text { Beta Monitor Sampler Radiation } \\
\text { Indicator in E/I Room E }\end{array}$ & & \\
\hline
\end{tabular}


PREOPERATIONAL TESTING - VENTILATION BUILDING VENTILATION SYSTEM

Revision No. 0

WHC-SD-W030-POTP-007

PAGE 18 OF 39

ATTACHMENT I - VENT BUILDING VENTILATION STACK MONITOR TEST PROCEDURE

\begin{tabular}{|c|c|c|c|c||}
\hline \multicolumn{5}{|c|}{ Appendix B - RWS Manual Valves Alignment } \\
\hline $\begin{array}{c}\text { VALVE } \\
\text { NUMBER }\end{array}$ & $\begin{array}{c}\text { REQUIRED } \\
\text { POSITION }\end{array}$ & INITIALS & IV \\
\hline \hline HV-AZ702-?? & $\begin{array}{l}\text { BETA Sample line manual isolation valve } \\
\text { in Monitor Panel in Stack Monitor Room. }\end{array}$ & OPEN & & \\
\hline HV-AZ702-?? & $\begin{array}{l}\text { Record Sample line manual isolation valve } \\
\text { in Monitor Panel in Stack Monitor Room. }\end{array}$ & OPEN & & \\
\hline & & & & \\
\hline & & & & \\
\hline & & & & \\
\hline & & & & \\
\hline & & & & \\
\hline & & & & \\
\hline & & & & \\
\hline & & & & \\
\hline
\end{tabular}

Performed

By
Verified

PRINT NAME INITIALS DATE

BY

PRINT NAME INITIALS DATE


PREOPERATIONAL TESTING - VENTILATION BUILDING VENTILATION SYSTEM

Revision No. 0

ATTACHMENT I - VENT BUILDING VENTILATION STACK MONITOR TEST PROCEDURE

\begin{tabular}{|c|c|c|}
\hline \multicolumn{3}{|c|}{ APPENDIX C - MANUAL VALVE CYCLE LIST } \\
\hline $\begin{array}{c}\text { Valve } \\
\text { Number }\end{array}$ & Valve Description & $\begin{array}{l}\text { Signature/ } \\
\text { Date/Time }\end{array}$ \\
\hline HV-AZ702-?? & $\begin{array}{l}\text { BETA Sample line manual isolation valve in Monitor } \\
\text { Panel in Stack Monitor Room. }\end{array}$ & \\
\hline HV-AZ702-?? & $\begin{array}{l}\text { Record Sample line manual isolation valve in Monitor } \\
\text { Panel in Stack Monitor Room. }\end{array}$ & \\
\hline & & \\
\hline & & \\
\hline & & \\
\hline & & \\
\hline & & \\
\hline & & \\
\hline & & \\
\hline & & \\
\hline & & \\
\hline & & \\
\hline & & \\
\hline & & \\
\hline & & \\
\hline & & \\
\hline & & \\
\hline & & \\
\hline
\end{tabular}


PREOPERATIONAL TESTING - VENTILATION BUILDING VENTILATION SYSTEM

Revision No. 0

WHC-SD-W030-POTP-007

PAGE 20 OF 39

ATTACHMENT I - VENT BUILDING VENTILATION STACK MONITOR TEST PROCEDURE

\begin{tabular}{|c|c|c|}
\hline \multicolumn{3}{|c|}{ APPENDIX D } \\
\hline STEP & PARAMETER & $\begin{array}{l}\text { STACK } \\
\text { FLOW }\end{array}$ \\
\hline & & 960 SCFM \\
\hline \multirow[t]{2}{*}{5.29 .6} & Fl-Display (SCFM) & \\
\hline & $\%$ Full Scale Stack Flow & \\
\hline 5.29 .7 & V/I-1 Channel 1 Ideal Output (mA) & \\
\hline 5.29 .8 & V/I-1Channel I Actual Output (mA) & \\
\hline 5.29 .10 & V/I-1 Channel $1 \%$ Deviation (:s2\%) & \\
\hline 5.29 .12 & Ideal Record and Beta Sample Flow (SCFM) & \\
\hline \multirow[t]{2}{*}{5.29 .13} & FI-AZ702KE-2 Display (SCFM) & \\
\hline & FI-AZ702K3-3 Display (SCFM) & \\
\hline 5.29 .15 & $\%$ Deviation Record Sample $(\leq 5 \%)$ & \\
\hline 5.29 .17 & $\%$ Deviation Beta Sample $(\leq 5 \%)$ & \\
\hline 5.29 .19 & V/I-1 Channel 2 Ideal Output (mA) & \\
\hline 5.29 .21 & V/I-1 Channel 3 Ideal Output (mA) & \\
\hline \multirow[t]{2}{*}{5.29 .22} & V/I-1 Channel 2 Actual Output (mA) & \\
\hline & V/I-1 Channel 3 Actual Output (mA) & \\
\hline 5.29 .24 & V/I-1 Channel $2 \%$ Deviation $(\leq 2 \%)$ & \\
\hline 5.29 .26 & V/I-1 Channel $3 \%$ Deviation $(\leq 2 \%)$ & \\
\hline \multirow[t]{2}{*}{5.29 .28} & TI-AZ702K3-1 Display $\left({ }^{\circ} \mathrm{F}\right)$ & \\
\hline & $\%$ Full Scale Stack Temperature & \\
\hline 5.29 .30 & V/I-2 Channel 2 Ideal Output (mA) & \\
\hline 5.29 .31 & V/I-2 Channel 2 Actual Output (mA) & \\
\hline 5.29 .33 & V/I-2 Channel $2 \%$ Deviation $(\leq 2 \%)$ & \\
\hline
\end{tabular}


PREOPERATIONAL TESTING - VENTILATION BUILDING VENTILATION SYSTEM

\begin{tabular}{|l|l|l|l|l|l|}
\hline \multicolumn{5}{|c|}{ APPENDIX E } \\
\hline \multicolumn{1}{|c|}{ STEP } & PARAMETER & $\begin{array}{l}1^{\text {sT }} \\
\text { CHECK } \\
\text { SOURCE }\end{array}$ & $\begin{array}{l}2^{\text {nd }} \\
\text { CHECK } \\
\text { SOURCE }\end{array}$ & $\begin{array}{l}3^{\text {rd }} \\
\text { CHECK } \\
\text { SOURCE }\end{array}$ & $\begin{array}{l}4^{\text {th }} \text { CHECK } \\
\text { SOURCE }\end{array}$ \\
\hline 5.30 .3 & Displayed Activity & & & & \\
\hline 5.30 .3 & V/I-2 Channel 1 Ideal Output $(\mathrm{mA})$ & & & & \\
\hline 5.30 .3 & V/I-2 Channel 1 Actual Output $(\mathrm{mA})$ & & & & \\
\hline 5.30 .6 & V/I-2 Channel 1 \% Deviation $(\leq 2 \%)$ & & & & \\
\hline
\end{tabular}


PREOPERATIONAL TESTING, VENT BUILDING VENTILATION \& STACK MONITOR WHC - SD - W030-POTP - 007

Revision No. ATTACHMENT I

PAGE 22 OF 39

Effective Date

\section{APPENDIX F}

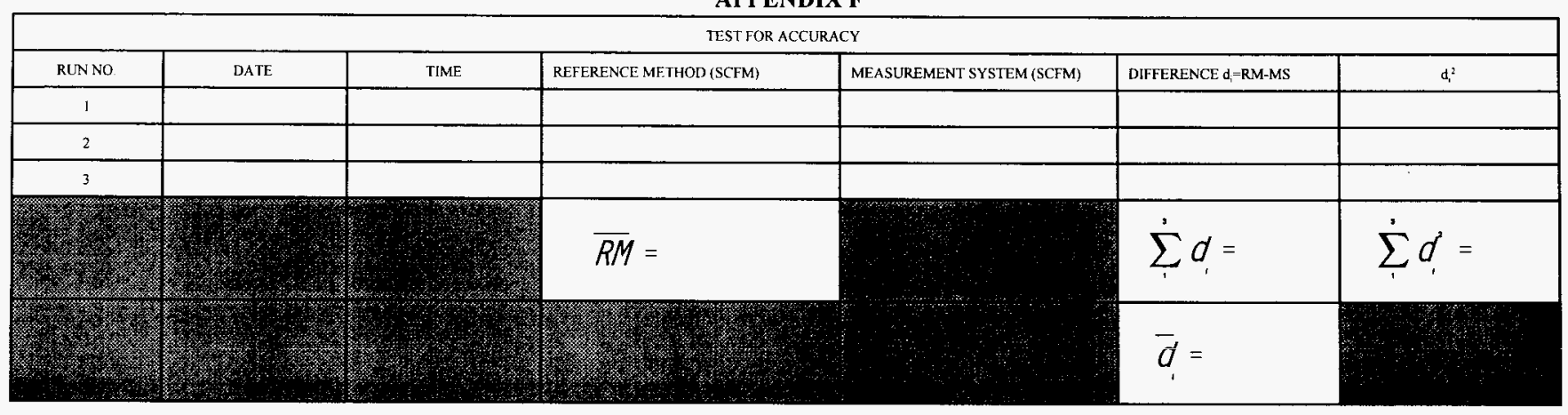

$\mathrm{Sd}=$

$\mathrm{cc}=$

$\mathrm{RA}=$ 
PREOPERATIONAL TESTING, VENT BUILDING VENTILATION \& STACK MONITOR WHC - SD-W030-POTP-007

\section{APPENDIX G}

\begin{tabular}{|c|c|c|c|c|c|c|c|c|c|c|}
\hline \multicolumn{11}{|c|}{ TEST FOR CALIBRATION DRIFT AND ZERO DRIFT } \\
\hline \multirow{2}{*}{ Run No. } & \multirow{2}{*}{ Date } & \multirow{2}{*}{ Time } & \multicolumn{2}{|c|}{ Zero Reading Volts } & \multicolumn{2}{|c|}{ Difference } & \multicolumn{2}{|c|}{ Calibration Reading Volts } & \multicolumn{2}{|c|}{ Difference } \\
\hline & & & Before & After & $\mathrm{d}_{\mathrm{i}}$ & $\mathrm{d}_{1}^{2}$ & Before & Affer & $d_{i}$ & $\mathrm{~d}_{1}^{2}$ \\
\hline 0 & & & 2.2 & & 6. & -2 & 2. & & (3) & 2 \\
\hline 1 & & & & & & & & & & \\
\hline 2 & & & & & & & & & & \\
\hline 3 & & & & & & & & & & \\
\hline & & & & & $\sum d=$ & $d$ & & & $\sum^{2} d=$ & $\sum^{3} d^{2}=$ \\
\hline & & & & & & & & & $\bar{d}=$ & \\
\hline
\end{tabular}

Sd:

cc:

Zero Drift:
$\mathrm{Sd}:$

cc:

Zero Drift: 
PREOPERATIONAL TESTING - VENTILATION BUILDING VENTILATION SYSTEM

Revision No. 0

WHC-SD-W030-POTP-007

PAGE 24 OF 39

ATTACHMENT I - VENT BUILDING VENTILATION STACK MONITOR TEST PROCEDURE

\begin{tabular}{|c|c|}
\hline \multicolumn{2}{|c|}{ APPENDIX H, 241-AZ-702 BLDG EXHAUST STACK SITE-SPECIFIC DATA } \\
\hline \multicolumn{2}{|r|}{ GENERAL INFORMATION } \\
\hline Stack: & $10^{\prime \prime}$ (circular diameter), approx. 16' tall \\
\hline Flow Test Ports: & $\begin{array}{l}\text { FTPs D and E, 3/4" half cplng w/plug, } \\
\text { height: approx. 6' (above work platform). }\end{array}$ \\
\hline Scaffolding: & Work platform installed for stack access. \\
\hline Exhaust Fan: & AZ702-K3-5-1A and AZ702-K3-5-1B; 1000 CFM @70º F \\
\hline HEPA Filter: & $\begin{array}{l}\text { AZ702-K3-4-1A and AZ702-K3-4-1B (each w/prefilter in series); } \\
\text { see H-2-131271 \& H-2-131073. }\end{array}$ \\
\hline \multicolumn{2}{|r|}{ REFERENCES } \\
\hline \multicolumn{2}{|c|}{$\begin{array}{l}\text { Include the following references if requested by Air Balance (AB), Operations (NPO), or Tank } \\
\text { Farm Power Operator (OP) personnel: }\end{array}$} \\
\hline \multicolumn{2}{|c|}{$\begin{array}{l}\text { H-2-131073, P \& ID Vent Bldg Ventilation System. } \\
\text { TO-060-350, Start, Stop and Operate 241-AZ-702 Building Ventilation System. }\end{array}$} \\
\hline \multicolumn{2}{|r|}{ PRECAUTIONS \& LIMITATIONS } \\
\hline \multicolumn{2}{|c|}{ No additional precautions \& limitations. See 6-TF-155, Section 4.0 . } \\
\hline & PREREQUISITES \\
\hline No additional pr & ee $6-\mathrm{TF}-155$, Section 6.0 \\
\hline
\end{tabular}


PREOPERATIONAL TESTING - VEN'TILATION BUILDING VENTILATION SYSTEM

Revision No. 0

WHC-SD-W030-POTP-007

PAGE 25 OF 39

ATTACHMENT I - VENT BUILDING VENTILATION STACK MONITOR TEST PROCEDURE

APPENDIX H, DATA SHEET 1

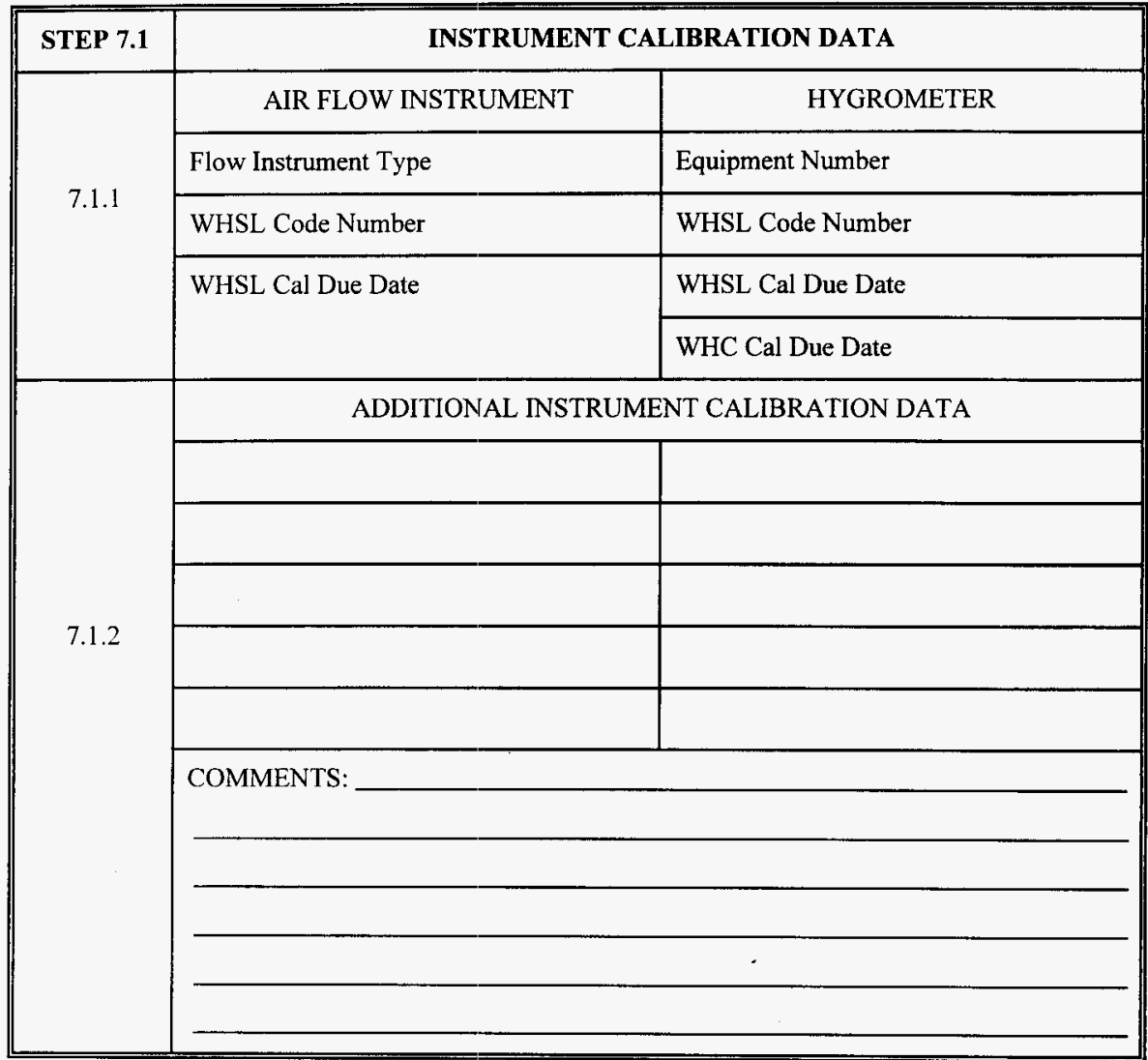

Initials/Date 
PREOPERATIONAL TESTING - VENTILATION BUILDING VENTILATION SYSTEM

Revision No. 0 WHC-SD-W030-POTP-007

PAGE 26 OF 39

ATTACHMENT I - VENT BUILDING VENTILATION STACK MONITOR TEST PROCEDURE

APPENDIX H, DATA SHEET 2

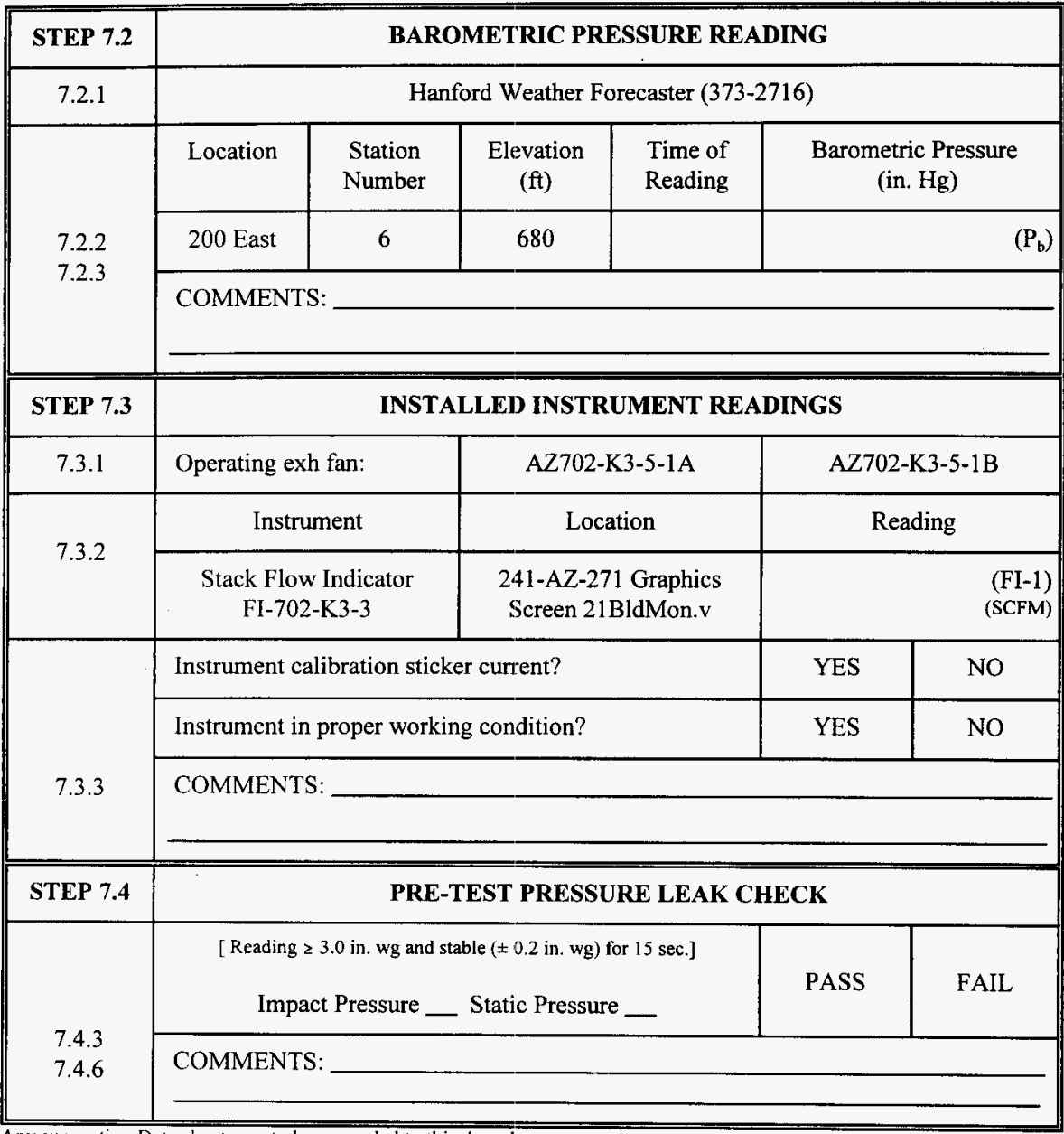

Any supporting Data sheets are to be appended to this data sheet.

Initials/Date 
PREOPERATIONAL TESTING - VENTILATION BUILDING VENTILATION SYSTEM

Revision No. 0

\begin{tabular}{|l|c|}
\hline \multicolumn{2}{|c|}{ 241-AZ-702 BLDG VENT } \\
\hline MEASUREMENT & TEST PORT \\
\hline Humidity & D \\
\hline Static Pressure & D \\
\hline Temperature & D, E \\
\hline Velocity Pressure & D, E \\
\hline Stack Diameter: & $10 \mathrm{in}$. \\
\hline Stock Areo: & $0.5454 \mathrm{sq} \mathrm{ft}$ \\
\hline Port Elevotion: & $703 \mathrm{ft}$ \\
\hline
\end{tabular}

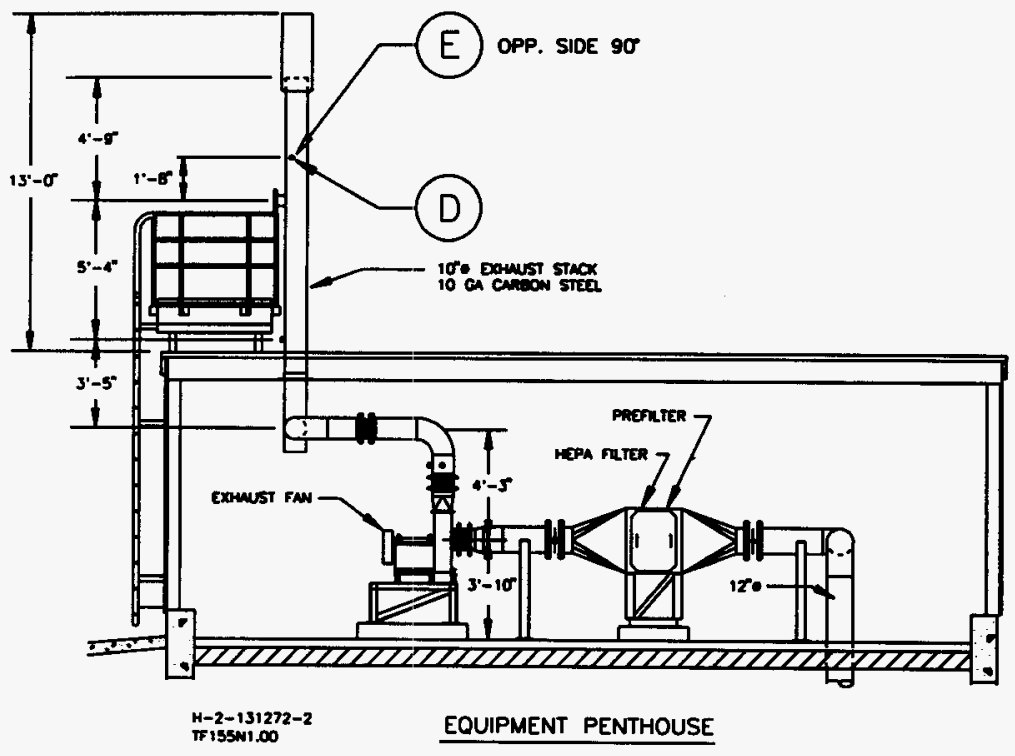

APPENDIX H, Figure 1. 241-AZ 702 Exhaust Stack 296-A-43 Air Flow Test Ports. 
PREOPERATIONAL TESTING - VENTILATION BUILDING VENTILATION SYSTEM

Revision No. 0

WHC-SD-W030-POTP-007

PAGE 28 OF 39

ATTACHMENT I - VENT BUILDING VENTILATION STACK MONITOR TEST PROCEDURE

APPENDIX H, DATA SHEET 3 (Sheet 1 of 2)

\begin{tabular}{|c|c|c|c|c|c|c|}
\hline \multirow[t]{8}{*}{ STEP7.5 } & \multicolumn{6}{|c|}{ STACK AIR FLOW MEASUREMENTS } \\
\hline & \multicolumn{6}{|c|}{ TEST PORT D } \\
\hline & \multicolumn{2}{|c|}{ Relative Humidity: } & \multicolumn{2}{|r|}{$(\mathrm{RH})$} & \multicolumn{2}{|c|}{ RETEST (IF REQUIRED) } \\
\hline & \multicolumn{2}{|c|}{ Static Pressure: } & \multicolumn{2}{|r|}{$\left(\mathrm{P}_{\mathrm{g}}\right)$} & \multicolumn{2}{|r|}{$\left(\mathrm{P}_{g}\right)$} \\
\hline & \multirow{2}{*}{$\begin{array}{c}\text { Traverse } \\
\text { Points* (in.) }\end{array}$} & \multirow{2}{*}{$\begin{array}{c}\text { Temp. } \\
\text { ts } \\
\left({ }^{\circ} \mathrm{F}\right)\end{array}$} & \multicolumn{2}{|c|}{ Velocity } & \multicolumn{2}{|c|}{ Velocity } \\
\hline & & & $\begin{array}{c}\text { VP } \\
\text { (in. wg) }\end{array}$ & $\begin{array}{l}\text { FPM }^{* *} \\
(\mathrm{ft} / \mathrm{min})\end{array}$ & $\begin{array}{c}\text { VP } \\
\text { (in. wg) }\end{array}$ & $\begin{array}{l}\text { FPM }^{* *} \\
(\mathrm{ft} / \mathrm{min})\end{array}$ \\
\hline & $1 / 2$ & & & & & \\
\hline & $1 / 2$ & & & & & \\
\hline \multirow[t]{2}{*}{7.5 .2} & $7 / 8$ & & & & & \\
\hline & $11 / 4$ & & & & & \\
\hline 7.5 .3 & $13 / 4$ & & & & & \\
\hline \multirow[t]{3}{*}{7.5 .4} & $21 / 4$ & & & & & \\
\hline & $27 / 8$ & & & & & \\
\hline & $33 / 4$ & & & & & \\
\hline \multirow[t]{8}{*}{7.5 .6} & $61 / 4$ & & & & & \\
\hline & $71 / 8$ & & & & & \\
\hline & $73 / 4$ & & & & & \\
\hline & $81 / 4$ & & & & & \\
\hline & $83 / 4$ & & & & & \\
\hline & $91 / 8$ & & & & & \\
\hline & $91 / 2$ & & & & & \\
\hline & $91 / 2$ & & & & & \\
\hline & TOTAL ts & & $\begin{array}{r}\text { TOTAL } \\
\text { FPM }\end{array}$ & & $\begin{array}{r}\text { TOTAL } \\
\text { FPM }\end{array}$ & \\
\hline
\end{tabular}

* Traverse points are measured relative to internal diameter (i.d.); none may be located within 0.5 in. of stack walls.

** ara anos vit

Any supporting Pitot Traverse Data sheets are to be appended to this data sheet.

Time test completed: Initials/Date 
PREOPERATIONAL TESTING - VENTILATION BUILDING VENTILATION SYSTEM

ATTACHMENT I - VENT BUILDING VENTILATION STACK MONITOR TEST PROCEDURE

APPENDIX H, DATA SHEET 3 (Sheet 2 of 2)

\begin{tabular}{|c|c|c|c|c|c|c|}
\hline STEP 7.5 & \multicolumn{6}{|c|}{ STACK AIR FLOW MEASUREMENTS } \\
\hline & \multicolumn{6}{|c|}{ TEST PORTE } \\
\hline & \multicolumn{4}{|c|}{ AIR FLOW MEASUREMENTS (CONTINUED) } & \multirow{2}{*}{\multicolumn{2}{|c|}{$\frac{\text { RETEST (IF REQUIRED) }}{\text { Velocity }}$}} \\
\hline & \multirow{2}{*}{$\begin{array}{c}\text { Traverse } \\
\text { Points* (in.) }\end{array}$} & \multirow{2}{*}{$\begin{array}{c}\text { Temp. } \\
\text { ts } \\
\left({ }^{\circ} \mathrm{F}\right)\end{array}$} & \multicolumn{2}{|c|}{ Velocity } & & \\
\hline & & & $\begin{array}{c}\mathrm{VP} \\
\text { (in. wg) }\end{array}$ & $\begin{array}{l}\mathrm{FPM}^{* *} \\
(\mathrm{ft} / \mathrm{min})\end{array}$ & $\begin{array}{c}\mathrm{VP} \\
\text { (in. wg) }\end{array}$ & $\begin{array}{l}\text { FPM** } \\
(\mathrm{ft} / \mathrm{min})\end{array}$ \\
\hline & $1 / 2$ & & & & & \\
\hline & $1 / 2$ & & & & & \\
\hline & $7 / 8$ & & & & & \\
\hline & $11 / 4$ & & & & & \\
\hline \multirow{3}{*}{7.5 .2} & $13 / 4$ & & & & & \\
\hline & $21 / 4$ & & & & & \\
\hline & $27 / 8$ & & & & & \\
\hline \multirow[t]{2}{*}{7.5 .3} & $33 / 4$ & & & & & \\
\hline & $61 / 4$ & & & & & \\
\hline \multirow[t]{2}{*}{7.5 .4} & $71 / 8$ & & & & & \\
\hline & $73 / 4$ & & & & & \\
\hline \multirow[t]{2}{*}{7.5 .5} & $81 / 4$ & & & & & \\
\hline & $83 / 4$ & & & & & \\
\hline \multirow[t]{4}{*}{7.5 .6} & $91 / 8$ & & & & & \\
\hline & $91 / 2$ & & & & & \\
\hline & $91 / 2$ & & (VP1) & & (VPI) & \\
\hline & TOTAL ts & & $\begin{array}{r}\text { TOTAL } \\
\text { FPM }\end{array}$ & & $\begin{array}{r}\text { TOTAL } \\
\text { FPM }\end{array}$ & \\
\hline
\end{tabular}

* Traverse points are measured relative to internal diameter (i.d.); none may be located within 0.5 in. of stack walls. **

Any supporting Pitot Traverse Data sheets are to be appended to this data sheet.

Time test completed: Initials/Date 
PREOPERATIONAL TESTING - VENTILATION BUILDING VENTILATION SYSTEM

Revision No. 0

WHC-SD-W030-POTP-007

PAGE 30 OF 39

ATTACHMENT I - VENT BUILDING VENTILATION STACK MONITOR TEST PROCEDURE

\section{APPENDIX H, DATA SHEET 4}

\begin{tabular}{|c|c|c|c|}
\hline STEP 7.6 & \multicolumn{3}{|c|}{ APPENDIX H, PITOT TUBE PERFORMANCE CHECK } \\
\hline \multirow[t]{2}{*}{$\begin{array}{l}7.6 .1 \\
7.6 .3 \\
7.6 .4\end{array}$} & \multicolumn{2}{|c|}{$\begin{array}{l}\mathrm{P}=\left[\left(\frac{\mathrm{VP} 1}{\mathrm{VP} 2}\right) \div-\mathrm{VP} 1\right. \\
\quad \\
\quad \text { If } \mathrm{P}> \pm 5 \% \underline{\mathrm{AND}} \mathrm{VP} 1<0.04 \text { in. wg, air flow retest is NOT required; } \\
\text { COG Engineer will determine acceptability of pitot tube performance. }\end{array}$} & PASS/FAIL \\
\hline & \multicolumn{3}{|l|}{ COMMENTS: } \\
\hline STEP 7.7 & \multicolumn{3}{|c|}{ K CHECK } \\
\hline \multirow[b]{2}{*}{$\begin{array}{l}7.7 .1 \\
7.7 .2\end{array}$} & \multicolumn{2}{|c|}{$\begin{array}{l}\text { [ Reading z } 3.0 \mathrm{in.} w g \text { and stable }( \pm 0.2 \mathrm{in.} \mathrm{wg} \text { ) for } 15 \mathrm{sec} \text {. ] } \\
\text { Impact Pressure __ Static Pressure }\end{array}$} & PASS/FAIL \\
\hline & \multicolumn{3}{|c|}{ COMMENTS: $\ldots \ldots \ldots+\ldots$} \\
\hline STEP 7.9 & \multicolumn{3}{|c|}{ STACK AIR FLOW CALCULATIONS } \\
\hline \multirow{3}{*}{7.9 .1} & \multirow{3}{*}{ Total $t s=t s 1+t s 2+t s 3+\ldots$} & Total Port D & $\overline{\text { (Sht 3) }}$ \\
\hline & & Total Port E & (Sht 3) \\
\hline & & ts $($ total $)(D+E)$ & \\
\hline 7.9 .2 & Average $t s=$ Total $t s \div 32$ & ts(avg) & \\
\hline 7.9 .3 & Velocity $(10 m)-4000, \sqrt{W r}$ & Data & et 3 \\
\hline \multirow{3}{*}{7.9 .4} & \multirow{3}{*}{ Total FPM $=$ FPM1 + FPM2 $+\mathrm{FPM}_{3}+\ldots$} & Total Port D & (Sht 3) \\
\hline & & Total Port E & (Sht 3) \\
\hline & & FPM(total) $(D+E)$ & \\
\hline 7.9 .5 & Average FPM $=$ Total FPM $\div 32$ & fpm(avg) & \\
\hline 7.9 .6 & Total CFM = Average FPM $\times 0.5454$ sq ft & cfm(total) & \\
\hline
\end{tabular}

Any supporting Data sheets are to be appended to this data sheet.

Initials/Date 
PREOPERATIONAL TESTING - VENTILATION BUILDING VENTILATION SYSTEM

Revision No. 0

WHC-SD-W030-POTP-007

PAGE 31 OF 39

ATTACHMENT I - VENT BUILDING VENTILATION STACK MONITOR TEST PROCEDURE

APPENDIX H, DATA SHEET 5 (Sheet 1 of 2)

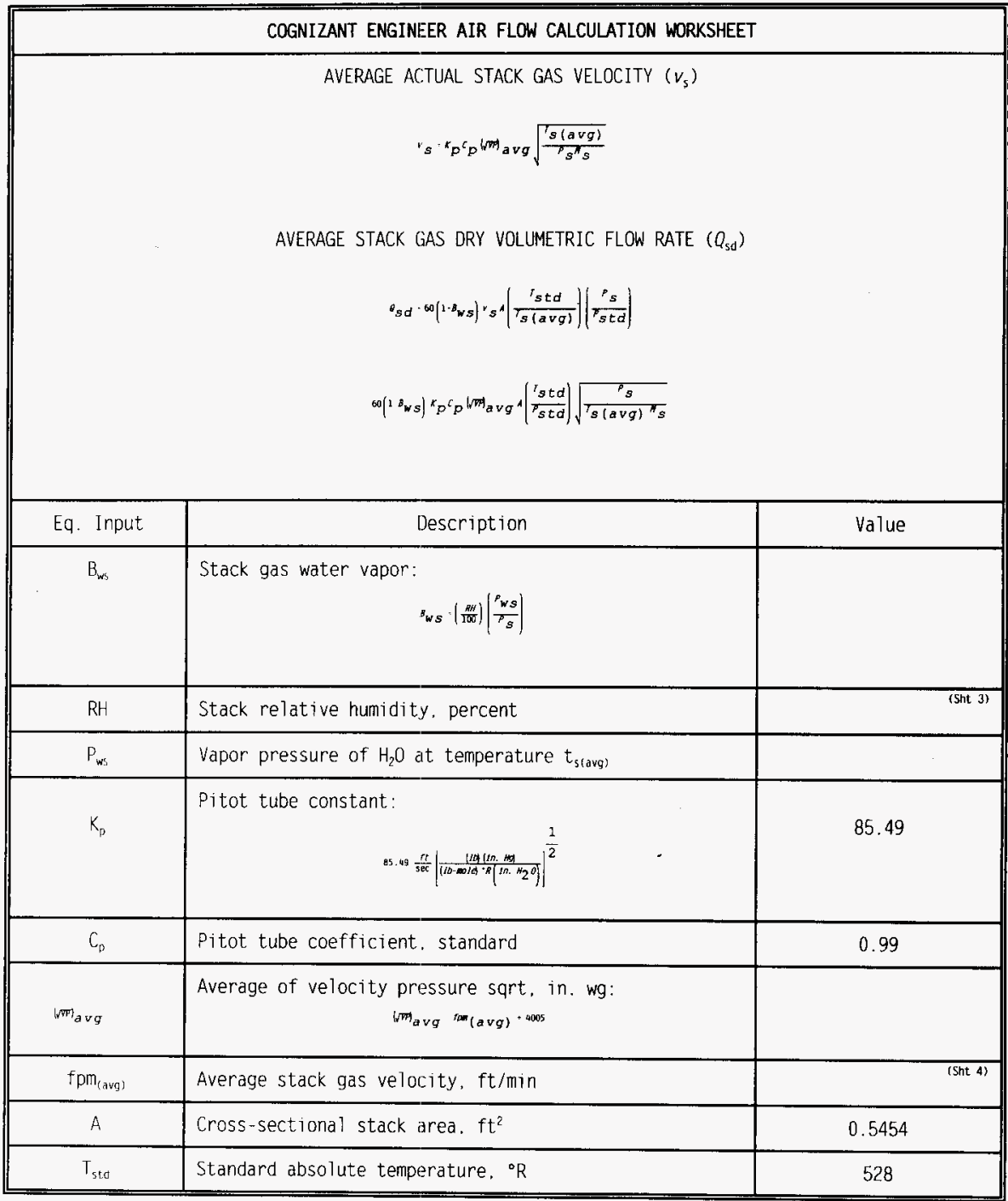

WHC-SD-W030-POTP-007 
PREOPERATIONAL TESTING - VENTILATION BUILDING VENTILATION SYSTEM

Revision No. 0

ATTACHMENT I - VENT BUILDING VENTILATION STACK MONITOR TEST PROCEDURE

APPENDIX $\mathrm{H}$, DATA SHEET 5 (Sheet 2 of 2)

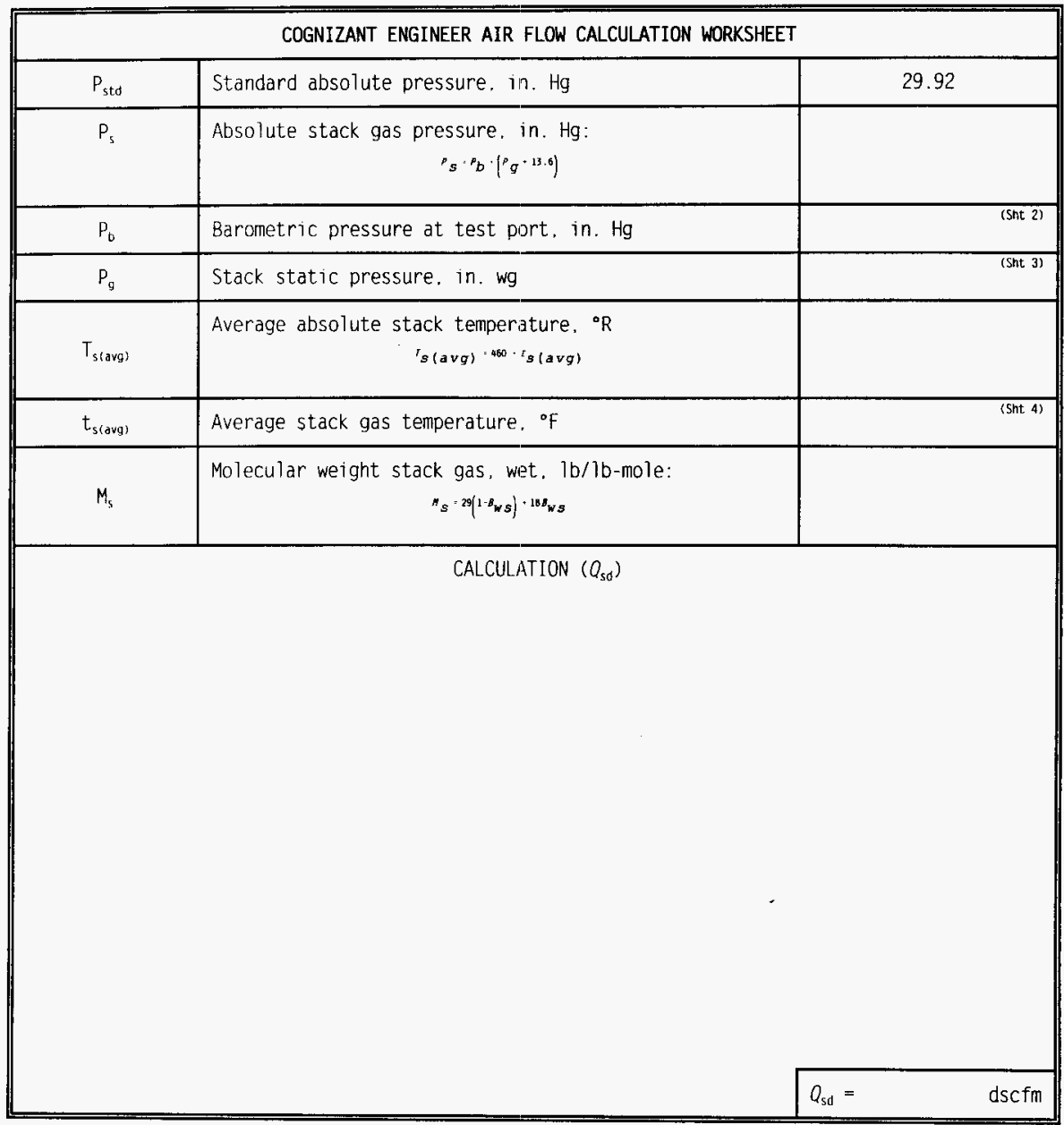

COG Engr Initials/Date: 
PREOPERATIONAL TESTING - VENTILATION BUILDING VENTILATION SYSTEM

Revision No. 0

WHC-SD-W030-POTP-007

PAGE 33 OF 39

ATTACHMENT I - VENT BUILDING VENTILATION STACK MONITOR TEST PROCEDURE

APPENDIX H, DATA SHEET 6

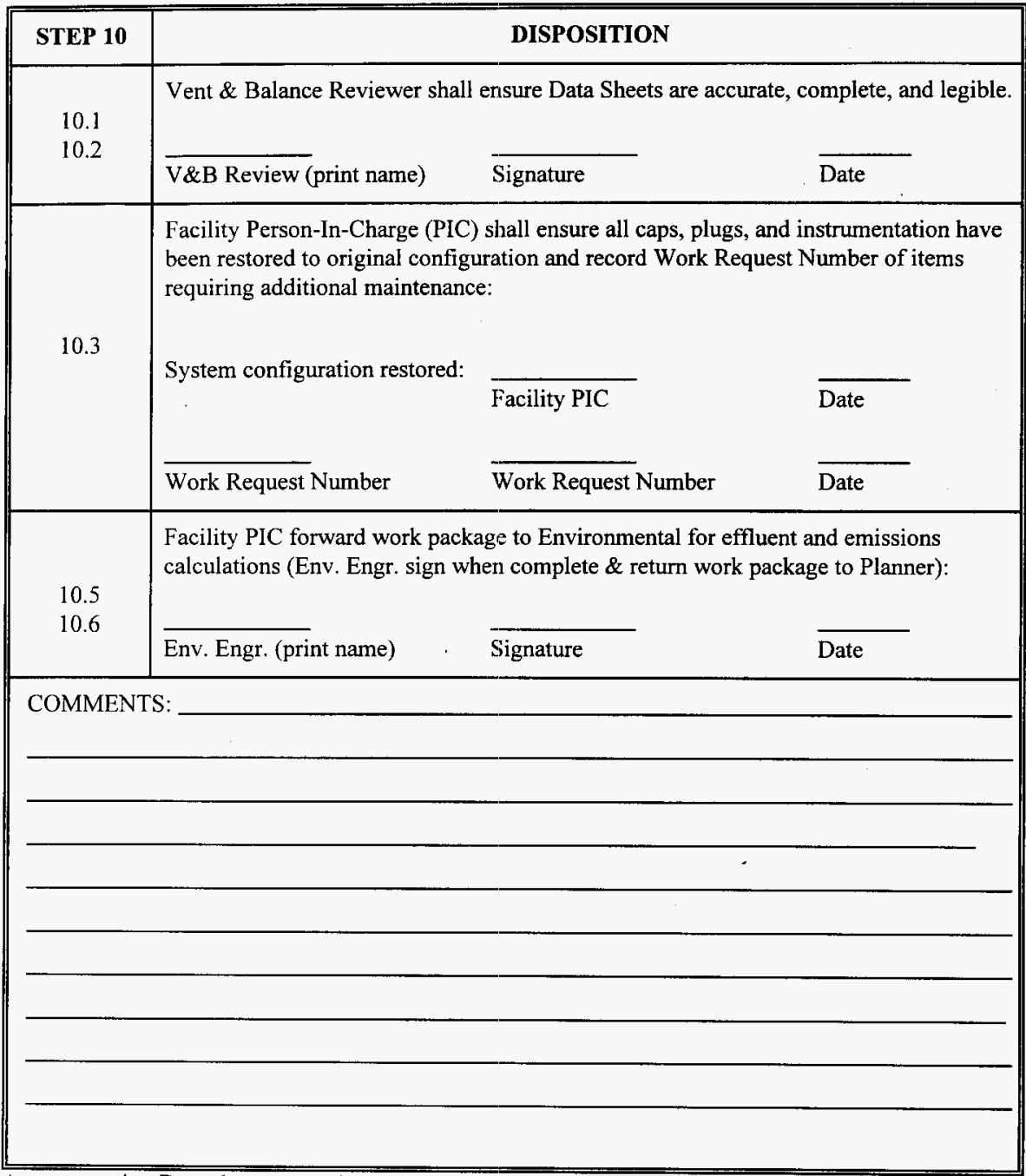

Any supporting Data sheets are to be appended to this data sheet. 
PREOPERATIONAL TESTING - VENTILATION BUILDING VENTILATION SYSTEM

Revision No. 0

WHC-SD-W030-POTP-007

PAGE 34 OF 39

ATTACHMENT I - VENT BUILDING VENTILATION STACK MONITOR TEST PROCEDURE

Effluent Engineering Worksheet (Sheet 1 of 2)

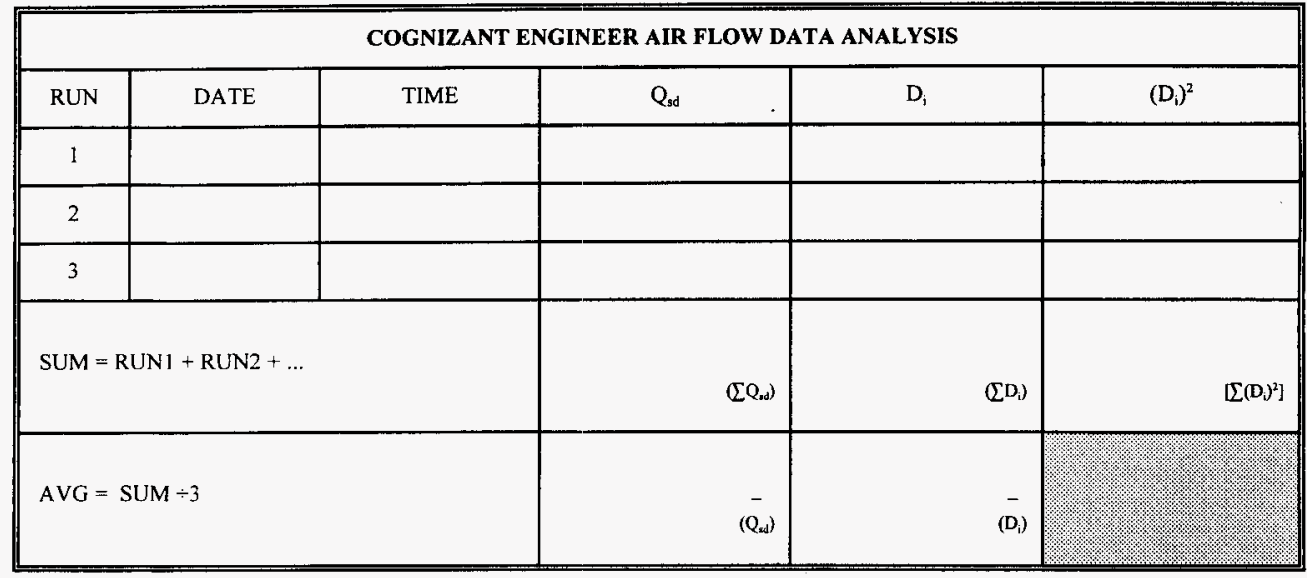

COG Engr Initials/Date: 
PREOPERATIONAL TESTING - VENTILATION BUILDING VENTILATION SYSTEM

Revision No. 0

WHC-SD-W030-POTP-007

PAGE 35 OF 39

ATTACHMENT I - VENT BUILDING VENTILATION STACK MONITOR TEST PROCEDURE

Effluent Engineering Worksheet (Sheet 2 of 2)

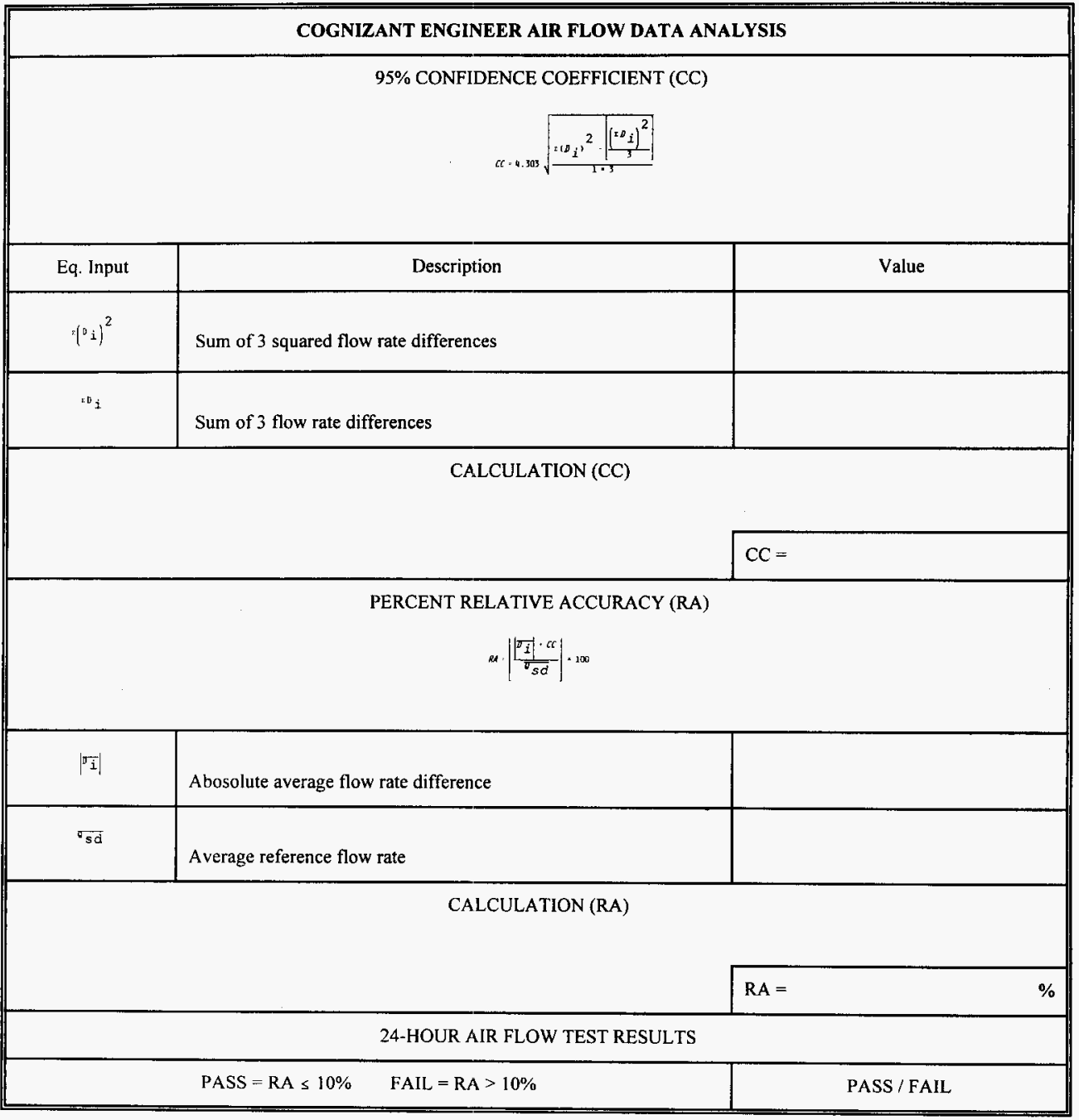


PREOPERATIONAL TESTING - VENTILATION BUILDING VENTILATION SYSTEM

Revision No. 0

WHC-SD-W030-POTP-007

PAGE 36 OF 39

ATTACHMENT J - SIGNATURE/INITIAL VERIFICATION

All persons involved in procedure performance, data recording, and verification or evaluation of test steps shall provide their name, job title, signature, and initials in the following table.

\begin{tabular}{|c|c|c|c|}
\hline NAME (PRINT) & TITLE & SIGNATURE & INITIAL \\
\hline & Test Engineer & & \\
\hline & $\begin{array}{l}\text { Control Room } \\
\text { Operator }\end{array}$ & & \\
\hline & Local Test Operator & & \\
\hline & Witness & & \\
\hline & Witness & & \\
\hline & Witness & & \\
\hline & & & \\
\hline & & & \\
\hline & & & \\
\hline & & & \\
\hline & & & \\
\hline & & & \\
\hline & & & \\
\hline & & & \\
\hline & & & \\
\hline
\end{tabular}




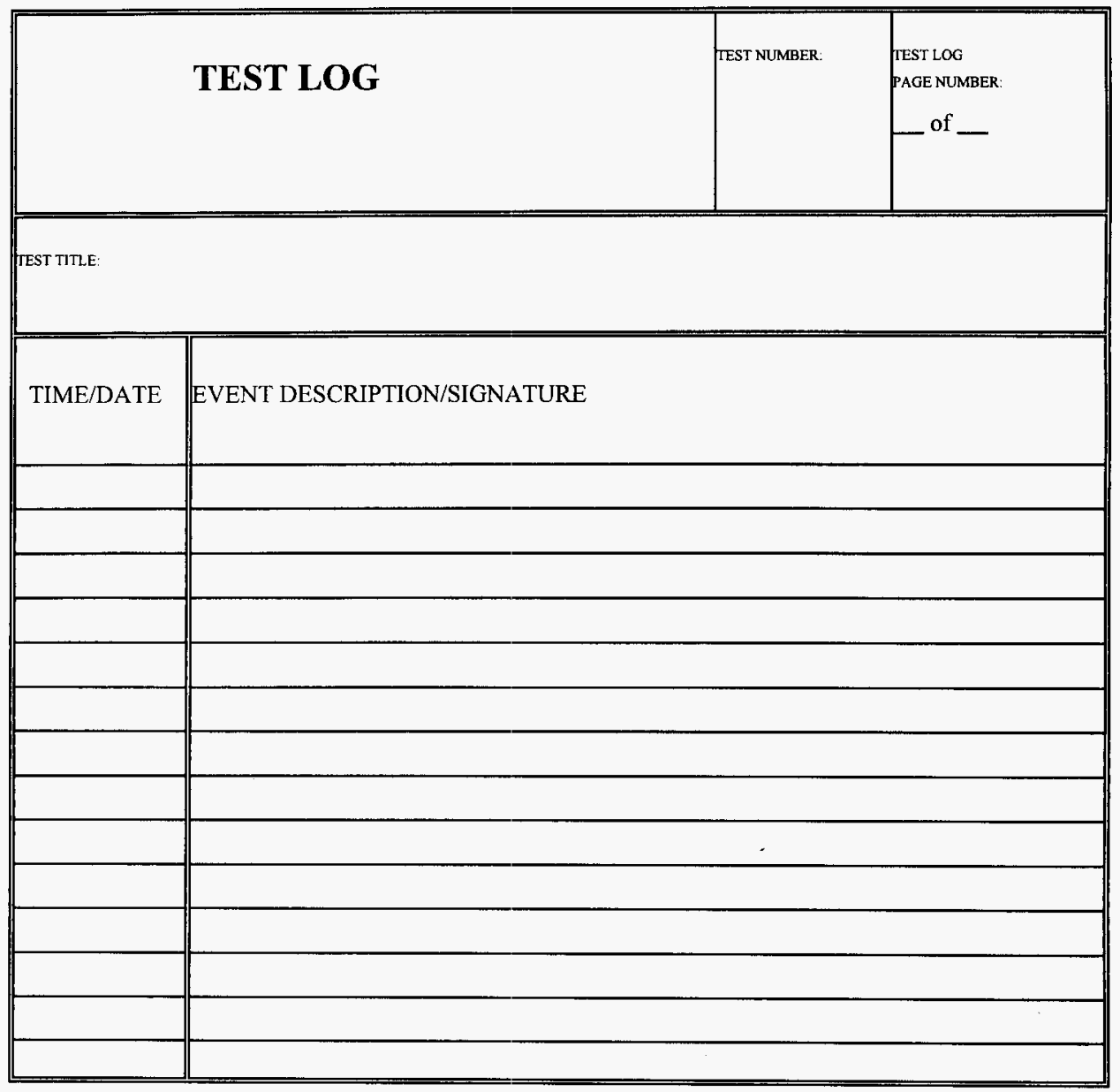


PREOPERATIONAL TESTING - VENTILATION BUILDING VENTILATION SYSTEM

Revision No. 0

WHC-SD-W030-POTP-007

PAGE 38 OF 39

ATTACHMENT L -TEST EXCEPTION REPORT

\section{TEST EXCEPTION REPORT}

\begin{tabular}{|l|l|l|}
\hline TEST PROCEDURE NO. \& SECTION: & TEST NAME: & T.E. NUMBER: \\
\hline
\end{tabular}

DESCRIPTION OF PROBLEM:

ORG:

DATE:

TE

DATE

DISPOSITION:

DISPOSITION AND RETEST REQUIREMENTS BY:

DATE

QAE CONCURRENCE WITH DISPOSITION (if required):
DISPOSITION ACTIONS COMPLETE:

Verified

By:

DATE

RETEST COMPLETE:

TE

DATE 
PREOPERATIONAL TESTING - VENTILATION BUILDING VENTILATION SYSTEM

Revision No. 0

WHC-SD-W030-POTP-007

PAGE 39 OF 39

ATTACHMENT M -TEST EXCEPTION LOG

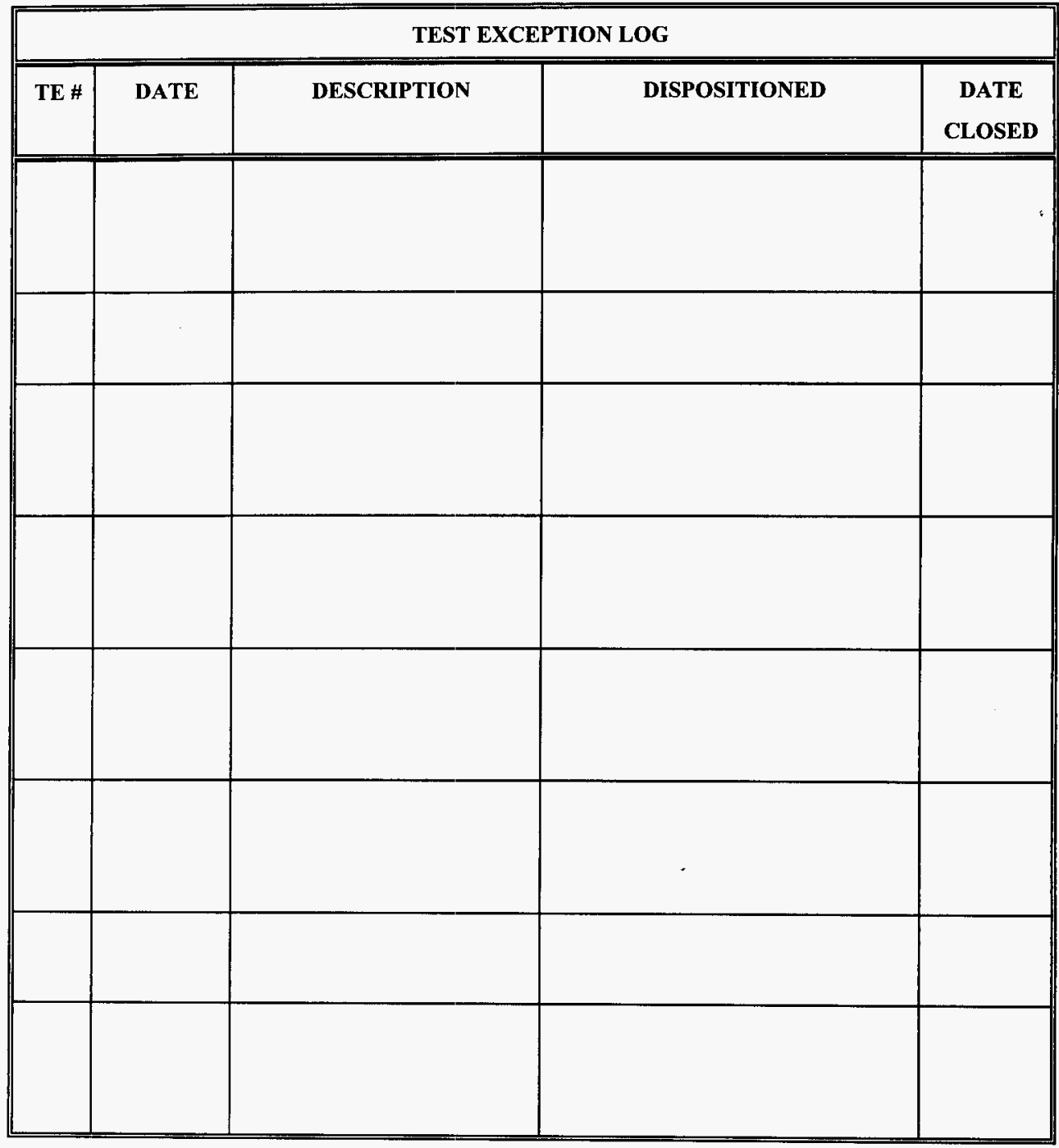




\section{DISTRIBUTION SHEET}

\begin{tabular}{|c|c|c|c|c|c|}
\hline \multirow{2}{*}{$\begin{array}{l}\text { To } \\
\text { Distribution }\end{array}$} & \multirow{2}{*}{\multicolumn{3}{|c|}{$\begin{array}{l}\text { From } \\
\text { Tank Farm Projects }\end{array}$}} & \multicolumn{2}{|c|}{ Page 1 of 1} \\
\hline & & & & \multicolumn{2}{|c|}{ Date } \\
\hline \multicolumn{4}{|l|}{ Project Title/Work Order } & \multicolumn{2}{|c|}{ EDT No. 616340} \\
\hline POTP-007 - TANK FARM VE & UPGRADE W030 & $8 K 240$ & & \multicolumn{2}{|c|}{ ECN No. -- } \\
\hline Name & MSIN & $\begin{array}{l}\text { Text } \\
\text { With All } \\
\text { Attach. }\end{array}$ & Text Only & $\begin{array}{l}\text { Attach./ } \\
\text { Appendix } \\
\text { Only }\end{array}$ & $\begin{array}{c}\text { EDT/ECN } \\
\text { Only }\end{array}$ \\
\hline
\end{tabular}

U.S. Department of Energy,

Richland Operations office

C. R. Pacheco

$57-52 \quad X$

ICF Kaiser Hanford Company

J. L. Henderson

E6-22

D. P. Devine

G3-12

Westinghouse Hanford Company

H. M. Chafin

F. T. Clifton (2 copies)

K. A. Colosi

D. B. Cole

K. A. Ealden

G. P. Hopkins

T. G. Howe1]

M. N. Islam

W. P. Nelson

G. L. Parsons

S. R. Pierce
G6-07

R3-25

R3-25

R3-25

R2-88

S5-03

G3-15

R3-08

R3-08

B4-08

S5-05
$\mathrm{X}$

$\mathrm{X}$

$x$

$x$

$x$

$x$

$x$

X

$x$

others

TFIC

Project Files

Central Files (orig. +2)

I A. Mahan (2 copies)

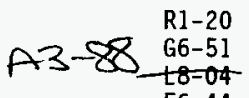

$X$
$X$
$X$
$X$ 